\title{
Comparative Anatomy of the Carotid-Basilar Arterial Trunk and Hindbrain Penetrating Arteries in Vertebrates
}

\author{
Sulman Rahmat* and Edwin Gilland
}

Howard University College of Medicine, 520 W St NW, Washington, DC 20059, USA

\begin{abstract}
Despite a long history of anatomical studies, the vascular organization of the brainstem is poorly known in most vertebrates. This review examines results from the past 150 years and compares them with a series of new vascular microdissections to provide an overall assessment of vertebrate hindbrain vasculature. The gross anatomy and embryology of cyclostomes, elasmobranchs, actinopterygians and tetrapods reveal a conserved suite of hindbrain vascular features including the: 1) origin and branching of cerebral carotids; 2) ontogenetic origins of the basilar artery from paired precursors, often followed by midline fusion to form an unpaired basilar; and 3) presence of a quasi-segmental series of transverse basilar artery branches that extend circumferentially around the brainstem giving off penetrating vessels. Phylogenetic analysis suggests that paired basilars are primitive for jawed vertebrates, with unpaired basilars arising independently in chondrichthyes, teleosts and tetrapods. Teleosts present a major exception to the general pattern, as penetrating arteries are restricted to a series of midline segmental basilar arterial stems that branch only within the neuroepithelium. This is clearly derived relative to all other vertebrates. Given the dissimilar body plans/lifestyles of groups served by the general brainstem vascular design, the conservation of adult hindbrain macrovasculature likely results from the similarity of embryonic cerebral blood supply across groups, rather than from convergence towards similar neurovascular functional designs. With the high degree of conservation in vertebrate brainstem neuronal anatomy, vascular patterning cues provided by the neuroepithelium practically assure that homologous brain regions are supplied by roughly similar arteries across taxa.
\end{abstract}

Keywords: Brainstem, vasculature, internal carotid artery, basilar artery, evolution.

\section{INTRODUCTION}

The anatomy and development of the vascular supply to specific brain regions and individual brain nuclei are partially documented in humans, but are largely lacking for most mammalian and non-mammalian model species. Given the radically different body types and habitats among fishes, amphibians and amniotes, it is expected that diverse anatomical and physiological strategies for brain vascularization would be employed in different taxa. Indeed, extensive structural diversity of 'loop' and 'network' cerebral microvasculature across vertebrates was described by Craigie and Scharrer [1,2]. Nevertheless, despite the variation in microanatomy, the gross macro-anatomy of vertebrate brainstem vasculature exhibits considerable conservation that was proposed by De Vriese in 1905 [3] to conform to a general plan based on shared developmental features. Reviews by Allis [4, 5] and Goodrich [6] showed that most of the variations at gross anatomical levels involve origination of cerebral vessels from different parts of the internal carotid, external carotid and post-cranial dorsal aorta.

A number of seminal papers have described the cerebral blood supply in various vertebrates, including hagfish by Müller [7], elasmobranchs by Hyrtl [8] and Allis [9], bony

*Address correspondence to this author at the Howard University College of Medicine, 520 W St NW, Washington, DC 20059, USA;

Tel: +1 202-806-6270; E-mail: sulman.rahmat@howard.edu fish by Grodzinski [10], frogs by Gaupp [11], reptiles by Rathke [12] and mammals by Hofmann [13], Padget [14], Duvernoy [15] and others. Surprisingly, the literature on more basal vertebrates has not been reviewed extensively and our survey shows that there is still much to be examined, both in terms of species sampled and in the overall depth of detail. For example, few of the studies on cranial arterial anatomy address brainstem vasculature other than indicating the origins of the basilar artery, and almost no studies in nonhuman vertebrates have attempted to integrate developmental, vascular and neuronal anatomy.

While some taxa have been examined in multiple studies to clarify certain points of vertebrate anatomy such as nomenclature of vessels (e.g., De Beer [16], Corrington [17]), many species have not been studied in-depth after their original descriptions. The lack of recent reviews of comparative hindbrain vascular anatomy stimulated us to survey the older literature and carry out a new series of gross anatomical experiments.

\section{Terminology}

In the $19^{\text {th }}$ century, diverse morphological hypotheses linking developmental and adult anatomies produced a confusing terminology of cerebral vessels, with numerous different names often being applied to the same vessel. Reviews by De Vriese [3], Hochstetter [18] and Allis [4] clarified most of the contentious issues and allowed subsequent workers (e.g., De Beer [16]; Corrington [17]) to develop tables of synonymy for carotid and cerebral vessels 
in different vertebrates. One critical step was establishing that the internal carotids in all taxa are formed, at least in part, as rostral extensions of the embryonic dorsal aorta [4]. Adult connections between internal carotids and paired dorsal aorta have been described in many different lower vertebrates [4, 7-10]. In groups where such connections are not present in adults (e.g., holocephalans, lungfish, amniotes), studies showed their loss during embryonic remodeling $[16,19]$. In most vertebrates, the paired internal carotids enter the hypophyseal fossa either as separate vessels, or after fusing to form a midline encephalic trunk (unpaired carotid a.). Human anatomical nomenclature refers to the vessels as "internal carotids" both before and after penetrating the dura mater. In studies of non-mammalian vertebrates, the term most commonly used for the main arterial stem piercing the dural sac has been "cerebral carotid" $[11,13,20]$. Because of the semantic problem of referring to the blood supply of the whole brain as "cerebral", many early authors used the technically more accurate term "encephalic" artery [21, 22]. Despite the questionable meaning, this review will use "cerebral carotid" artery instead of "encephalic" due to the widespread acceptance and familiarity of the former. The cerebral carotids generally travel through the hypophyseal fossa to bifurcate near the hypothalamus and optic nerve into two divisions, usually called "anterior and posterior rami of the cerebral carotid artery" $[11,13,20,23,24]$. For the sake of brevity, these will be referred to as the anterior and posterior cerebral carotid arteries (ACCs and PCCs, respectively; Fig. 1). The ACCs generally give rise to branches that supply the diencephalon and telencephalon. The PCCs extend caudally along the sides of the midbrain and either fuse in the midline to form a single basilar artery, or remain as paired vessels running under the hindbrain. The frequent use of "posterior cerebral artery" for major portions of the PCCs in basal vertebrates is particularly inappropriate, since, as shown by De Vriese [3], Padget [14], and Moffat [25], the mammalian posterior cerebral artery is a late-forming collateral of the PCCs and cannot be homologous to the main stem of the latter in other groups (see below).

Other branches of the internal carotid arteries also have numerous synonyms [16, 17]. The major branch generally arising just prior to the entry of the internal carotids into the hypophyseal fossa and extending rostrally to supply the maxillary, orbital and nasal regions in basal vertebrates is frequently called the "orbital" [16], "orbitonasal" [21], "palatine" [11] or "facial carotid" [26] artery. The former two terms have been used frequently for cartilaginous and bony fish, while the latter two are widely used in tetrapods. This vessel has also been named erroneously as "external carotid" in numerous studies $[17,27]$. The external carotids in mammals are clearly extensions from the ventral portions of the pharyngeal aortic arches that subsequently join the carotid stems during later development [6]. This term should therefore be avoided for any vessels branching from the internal carotids in lower vertebrates. The artery branching from the efferent pseudobranchial artery (when present) or directly from the internal carotid and supplying the choroidal layer of the eye will be referred to here as the ophthalmic artery [16; ophthalmica magna of Müller, 7]. The branch arising near the separation of anterior and posterior cerebral carotids and traveling along the optic nerve to the neural retina will be called the optic artery [16; ophthalmica minor of Müller, 7; central retinal arteries of others]. Classic reviews such as those by Hochstetter [18] and Goodrich [6] outlined the basic homologies of the orbital, ophthalmic and optic arteries across vertebrates. However, the diversity of origins, areas of supply, anastomoses and substitution by neighboring vessels through ontogenetic "capture" means that establishing detailed homologies for these arterial stems will require a large-scale review and are not within the scope of the present survey.

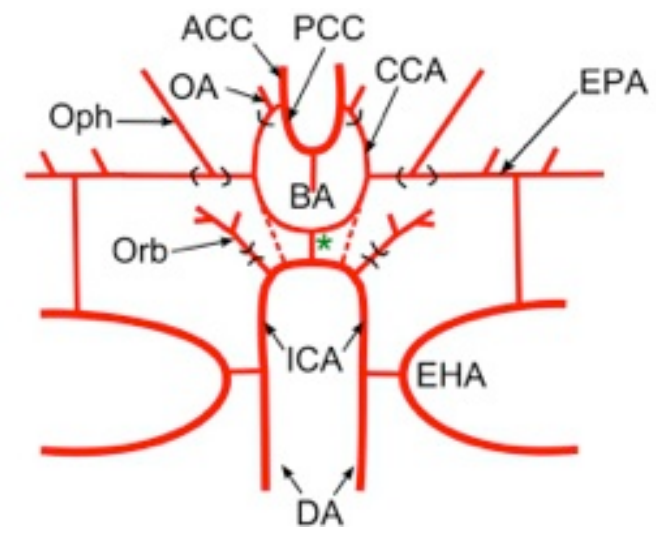

Fig. (1). Terminology of Main Cerebral Arterial Branches: Schematic diagram modified after Allis [9] showing main stems and branches of the internal carotid arterial pathways typical for vertebrates. Vessels supplying the internal carotid (ICA) vary widely among vertebrates but primitively include the paired dorsal aorta (DA) and efferent vessels of the hyoid (efferent hyoid artery, EHA) and mandibular arches (efferent pseudobranchial arteries, EPAs). Distribution branches supplying structures outside of the braincase typically include an orbital artery (Orb) branching from the extracranial internal carotid and an ophthalmic artery (Oph) branching from the EPAs (or equivalent). The cerebral carotids (CCAs) arise either as continuations of the paired dorsal aorta (dashed lines) or by bifurcation of a midline, unpaired carotid (= encephalic) trunk (green *). The cerebral carotids divide into anterior (ACCs) and posterior (PCCs) divisions, with an optic artery (OA) arising either immediately before or after the branching. The PCCs either meet in the midline beneath the rostral hindbrain to form an unpaired basilar artery (BA), or they continue caudally as paired basilar (rhombencephalic) vessels.

\section{Penetrating Arteries and 'Loops' Versus 'Nets'}

Major branches of the intradural carotid and basilar arteries travel along the pial surface of the brain and give off smaller-caliber vessels that pierce the pia and enter the brain parenchyma as "penetrating arteries." In most vertebrates, the major vessels branch extensively outside the pia before giving off penetrating branches. The penetrating arteries generally course towards the ventricular surface, often along direct radial paths. In contrast, the penetrating arteries supplying the brainstem in teleost fishes are generally restricted to a small number of large stems arising from the PCCs and basilar arteries that penetrate at or near the ventral midline of the mid- and hindbrain. Arterial branching occurs primarily outside the brain wall (extramural) in most vertebrates but, uniquely, it occurs almost entirely within the brainstem (intramural) in teleosts. Classic studies by Craigie [1], Scharrer [2] and others showed that within the brain parenchyma, penetrating arteries either form 1) sharp hairpin 
turns which lead back to the pial surface as unbranched venous channels (loops) or 2) extensive anastomotic connections with neighboring penetrating vessels (nets). In the first instance, each penetrating vessel is terminal, having no communication with other vessels, a pattern seen in various lampreys, amphibians, reptiles and marsupials [1, 2, 28]. The contrasting network-type microvasculature with many anastomoses in a dense capillary bed is seen in a broader group of vertebrates, including hagfish, chondrichthyes, and most osteichthyes, amphibians, reptiles, birds and mammals. Although the phylogeny of brain microvasculature has not been worked out in detail, it seems clear that loop and network patterns have evolved from one another multiple times within vertebrates $[1,2]$. As shown below, the presence of 'loop' or 'net' microvascular patterns appears to have little influence on the general macroscopic vascular anatomy present in any given group of vertebrates.

Overall, this review outlines general brainstem macrovascular patterns by examining anatomical and embryological data from most major vertebrate clades (Fig. 2, tree). The results will highlight design similarities between groups as well as unique features found only within specific taxa. In addition to noting the stems and proximal branches of the cerebral carotid arteries in these groups, two characteristics of hindbrain vasculature will be examined across species: the presence of paired versus unpaired basilar arteries and the pattern of transverse and median penetrating arteries. The discussion will present evidence for and against the hypothesis that paired basilars are primitive for all vertebrates and that a single basilar arises three times independently in cartilaginous fishes, teleosts, and tetrapods. The lack of circumferential transverse arteries and restriction of penetrating branches to midline central arteries in teleosts will be proposed as a unique evolutionary innovation linked to the taxonomic success of this group.

\section{MATERIALS AND METHODS}

\section{Dissection of Adult Vertebrate Cerebral Vasculature}

Cranial and cerebral vascular anatomies were examined in vascular-injected, commercially prepared (Carolina Biological, Burlington, NC) specimens of lamprey (Petromyzon marinus, $\mathrm{N}=6$ ), perch (Morone americana,

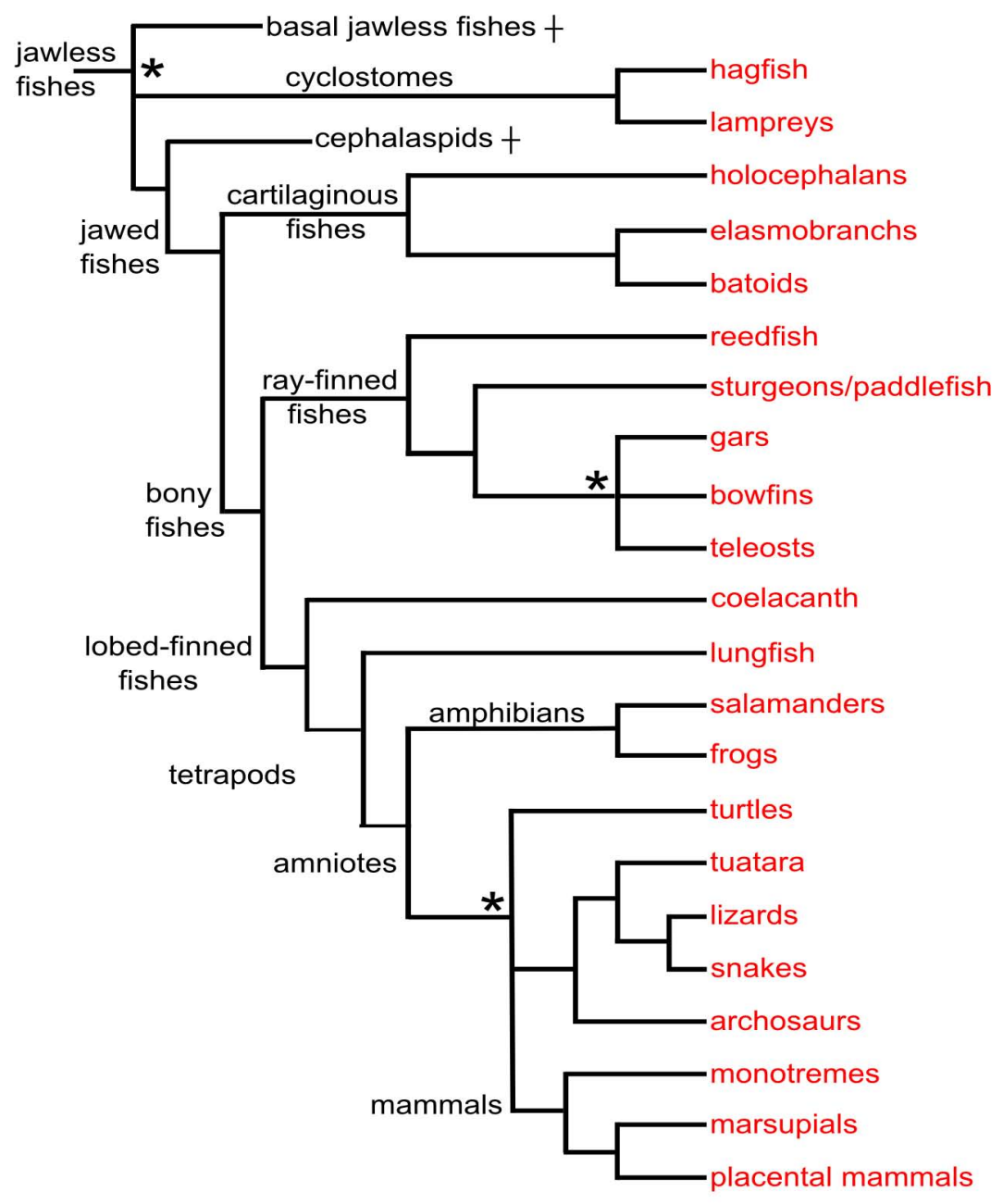

Fig. (2). Cladistic relationships of the taxa covered in this study. Relationships are based on Janvier [34], Delarbre et al. [35] and the Tree of Life Web Project (www.tolweb.org), with informal names substituted for most groups. Nodes for which there is no general consensus are left unresolved (indicated by asterisks). Some taxa not mentioned in the study are omitted, e.g., caecilians. Two wholly extinct agnathan groups are included: basal jawless fishes, a diverse assemblage of primitive vertebrates, and cephalaspids. 
$\mathrm{N}=6$ ), spiny dogfish (Squalus acanthias, $\mathrm{N}=7$ ), bull frog (Rana catesbiana, $\mathrm{N}=6$ ) and painted turtle (Chrysemys picta, $\mathrm{N}=2$ ). Preserved specimens of reedfish (Calamoichthys $s p$., $\mathrm{N}=1$ ), bowfin (Amia calva, $\mathrm{N}=1$ ) and gar (Lepisosteus osseus, $\mathrm{N}=1$ ) that did not have vascular injections were also dissected. Specimens were dissected using a stereo dissecting microscope and photographed using a Nikon Coolpix 4500 camera mounted on the microscope.

\section{Analysis of Serially Sectioned Material}

The relationship between ponto-medullary transverse branches and adjacent rhombomeric borders was examined in a closely spaced developmental series of sectioned human embryos (Carnegie Collection, Armed Forces Institute of Pathology, Bethesda, MD). These paraffin-embedded series cut in all three planes provided the basis for the classic study of human cranial arterial development by Padget [14]. Largecaliber vascular channels were examined in serial sections of uninjected adult brains of goldfish (Carassius auratus). These specimens had reticular and/or motor neurons retrogradely labeled with biocytin and were prepared for previous studies [methods in 29]. Sections were photographed with a Zeiss AxioCam HR mounted on an AxioPlan microscope. Image processing was performed with Zeiss AxioVision and NIH ImageJ software. Figures were prepared using Photoshop and Illustrator (Adobe).

\section{Sources of Scientific Literature}

Comparative studies of brain vasculature are scattered throughout the literature of animal morphology, veterinary and human anatomy, neurosurgery, paleontology and developmental biology. The older literature was mined starting with major early twentieth century reviews and extended through reference lists in the original studies. The age and rarity of many of the studies required the use of major research libraries including the Smithsonian National Museum of Natural History and the Marine Biological Laboratory (Woods Hole) and online resources such as Archive.org, Biodiversity Heritage Library and the Hathi Trust. Additionally, since many of the older papers were written in Italian, German, and French, rough translations of non-English articles were prepared using Google translator, before being corrected with the assistance of native speakers.

\section{RESULTS}

\section{Conserved Development of Brainstem Arterial Supply in Vertebrates}

At the gross anatomical level, origination of cerebral vessels from different parts of the internal carotid, external carotid and post-cranial dorsal aorta has been described for numerous groups $[1,3-5,14,20]$. Likewise, the microvasculature that serves to distribute blood within the brain varies between 'loop' and 'network' architectures, both within and among various higher taxa $[1,2]$. However, despite the significant diversity in adult brain vasculature, a highly conserved framework of brainstem vessels is evident when comparing the embryonic development of distantly related vertebrates such as dogfish [17, 30], zebrafish [31] and human [14].

In these species, the primitive internal carotid artery and a pial angiogenic network beneath the hindbrain neuroepithelium contribute to form a carotid-basilar arterial tree (Fig. 3). The dogfish, zebrafish, and human internal carotid arteries (ICAs) originate as rostral extensions of the paired dorsal aorta anterior to the dorsal ends of the hyoid aortic arches. The paired ICAs in dogfish embryos fuse in the midline caudal to the hypophyseal region to form an elongated, unpaired ICA termed as the "sinus cephalicus", which develops into the adult encephalic trunk (see below). Within the hypophyseal 'fossa', the unpaired ICA divides into paired cerebral carotid arteries (CCA). Similarly, the primitive ICAs in embryonic humans and zebrafish follow the same course through the hypophyseal region and continue as the paired CCA. The primitive ICAs in humans remain paired throughout development, while those in zebrafish unite in the midline at later stages, to form an unpaired ICA similar to, but likely not homologous to, that in sharks. In all these groups, the paired CCA approach the forebrain-midbrain junction on either side of the hypophysis, between the stalks of the optic vesicles and the oculomotor nerve roots, and branch into anterior and posterior divisions (ACCs and PCCs; Fig. 3). The ACCs supply most of the forebrain in these species, through a pair of main olfactory arterial trunks that give off dorsal and ventral branches to the diencephalon and telencephalon. The PCCs turn sharply caudally and extend along the ventrolateral sides of the midbrain where they become continuous with bilateral "neural arteries" (BNA; Fig. 3) that are coalescing from scattered angiogenic cells beneath the hindbrain neuroepithelium, just medial to the roots of the cranial nerves. The angiogenic cells contributing to the BNA, and to the eventual basilar artery which forms from them, have been shown to migrate medially from laterally located primordial hindbrain venous channels in both humans [14] and zebrafish [32,33]. In zebrafish, the contribution of vasculogenic cells from the lateral venous channels appears as a series of paired vascular arches that invade the hindbrain neuroepithelium and connect to the basilar artery anlage coalescing in the midline beneath the hindbrain $[32,33]$. The paired longitudinal vessels formed by the PCCs and BNAs fuse in the midline, to greater or lesser degrees, to form the basilar artery (BA). The axial level of this fusion varies during development and within adult populations for all three of these species, but is generally located between the levels of the oculomotor and trigeminal nerve roots, beneath the caudal midbrain (human) or rostral hindbrain (dogfish, zebrafish). Midline fusion does not occur in some vertebrates, resulting in direct formation of "paired basilar arteries" from the paired PCC-BNA rudiments.

An extensive series of arteries serving the diencephalon, mesencephalon and rhombencephalon branches off from both the paired and unpaired portions of the PCC/BA in sharks and humans. In general, branches supplying the diencephalon and mesencephalon arise from the paired PCCs, while those supplying the cerebellum and hindbrain arise from the BA. The rhombencephalic vessels appear to closely match the rhombomeric pattern of the neuroepithelium. In humans, sharks and most other vertebrate groups, these arteries circle the pial surface of the brain, sending penetrating branches into the brain wall along their courses. The basilar artery and transverse branches that become cerebellar and pontine arteries are well-established in $9 \mathrm{~mm}$ embryos (Fig. 3). In some vertebrates, such as 

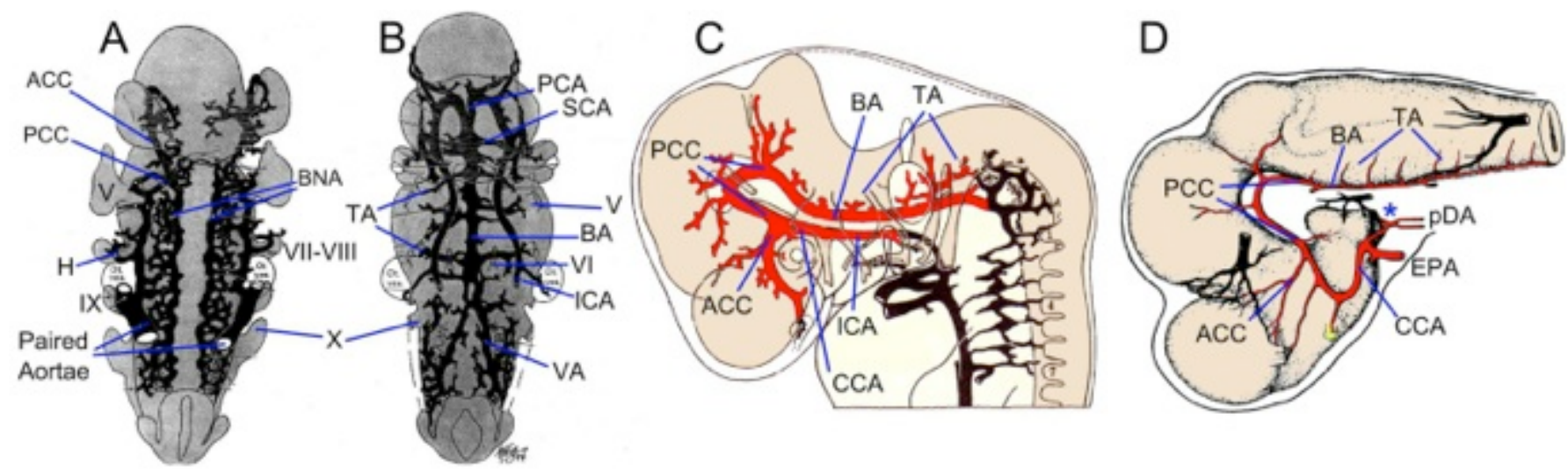

Fig. (3). Development of Carotid-Basilar Axis in Human and Dogfish A-B. Graphic reconstructions of ventral views of the brain and cranial arteries in Stage 13 (4mm; A) and Stage 16 (9mm; B) human embryos, modified from Padget [14]. By 4mm, the internal carotid artery (ICA) has already divided into cranial (anterior; ACCs) and caudal (posterior; PCCs) divisions. Note that the bilateral neural arteries (BNAs) and adjacent pial plexus reorganize between these two stages to form a vertebro-basilar system that has the same basic topography as in adults. C. Lateral view of reconstructed 9mm (stage 16) human embryo modified from Padget [14] showing the cerebral carotid artery (CCA) passing caudal to the optic vesicle and dividing into ACCs and PCCs. The basilar artery (BA) has been formed from the fusion of the BNA and already is giving off transverse arterial branches (TA). D. Lateral view of the brain and carotid branches of a $32 \mathrm{~mm}$ embryo of Squalus acanthias modified from Sterzi [45]. The paired dorsal aorta (pDA) join to form an unpaired "encephalic" trunk (asterisk) which then enters the hypophyseal fossa and divides into left and right cerebral carotids (CCAs) which are joined by the efferent pseudobranchial arteries (EPAs). The CCA divides into ACC and PCC divisions, with the latter curving caudally beneath mid and hindbrain where they fuse in the midline to join the BA. Cranial nerve roots are indicated by roman numerals as H, hyoid artery; PCA, posterior cerebral artery; SCA, superior cerebellar artery; VA, vertebral artery.

sharks, the basilar artery is continuous with a ventral spinal artery that runs beneath the spinal cord. In other groups, such as humans and zebrafish, the basilar artery bifurcates caudally to connect with paired longitudinal vessels formed by the fusion of earlier intersomitic spinal segmental vessels $[14,30]$. In humans, these contributions from cervical and spinal dorsal intersegmental vessels give rise to the vertebral arteries [19], which are likely not homologous to the spinal vessels that join the basilar artery in zebrafish. In most vertebrates, an additional series of vessels connecting the PCC/basilar arteries ventrally with the paired dorsal aorta has been described. These, often transitory, connecting arteries are closely associated with the roots of cranial nerves and in mammalian embryos are known as the primitive trigeminal, stapedial and hypoglossal arteries $[14,19]$.

\section{Jawless Fishes (Agnatha)}

Agnathans comprise a diverse group of basal vertebrates, most of which are extinct taxa known only from fossils [34]. Lampreys and hagfish are the only extant jawless fishes, and although they differ greatly in anatomy, they are currently grouped together as Cyclostomata based mainly on molecular phylogenetic data [35]. Although the brains of lampreys and hagfish are "similar" in lacking some basic features found in all jawed vertebrates, such as a well developed cerebellum and ventricular system, unique 'cyclostome' features are not clearly evident. In contrast, cephalaspids, an extinct group known from many extremely well preserved fossil specimens, appear to share numerous features, both skeletal and inferred neural ones, with both lampreys and jawed vertebrates, thus providing a plausible phylogenetic link between these groups [34].

\section{Cerebral Vascular Pattern in Cyclostomes}

Cerebral vascular supply has been examined in adult hagfish (Eptatretus cirrhatus [7]; E. stouti [36]; Myxine glutinosa [37]) and larval [38] and adult [39] marine lampreys (Petromyzon marinus). Numerous differences in adult vascular anatomy between hagfish and lamprey make it difficult to identify a common pattern within cyclostomes. In both groups, the brain is supplied by paired vessels ("common carotids") that extend rostrally from the efferent branchial/dorsal aorta arterial system and then give off one or more branches to lingual, orbital, and nasal regions outside the braincase (Orb, Fig. 4A). The branching pattern and location of entry into the hypophyseal fossa of the cerebral carotids differ considerably between these groups and neither closely matches the general gnathostome pattern. The orbital arteries of hagfish ("external carotid" of Müller) pass bilaterally forward along the outside of the basal plate giving off branches to the dental apparatus, lateral walls of the skull and the muscles and skin of the tentacular region surrounding the mouth [36]. The right and left internal carotid arteries of hagfish fuse to form a midline arteria carotis interna impar (= arteria vertebralis impar; Müller [7]) that runs forward a short distance beneath the notochord before dividing into paired cerebral carotid arteries (CCA; Fig. 4A). This median unpaired arterial trunk is similar to the unpaired internal carotid in cartilaginous fish (see below), but enters the braincase near the otic capsule rather than in the hypophysial region as in gnathostomes. This difference could result from the extreme cerebral flexure in hagfish which places the infundibulum beneath the rostral hindbrain [40]. The internal carotids in lampreys, which have no median trunks or commissural branches, divide outside the 
braincase into anterior (ACCs) and posterior (PCCs) cerebral carotid arteries (Fig. 4B). These enter the braincase separately [39] in a manner not seen in other adult vertebrates. Within the dura, the branching patterns and brain regions supplied by the ACCs and PCCs in hagfish and lampreys are similar to the general gnathostome developmental pattern described above [37, 39]. Just behind the optic nerves, the ACCs divide into dorsal and olfactory branches that supply the forebrain. While the PCCs in most gnathostomes fuse along the midline of the hindbrain to form a single basilar artery just cranial to $\mathrm{CN} \mathrm{V}$, in adult cyclostomes they continue as paired basilars, a condition seen only sporadically among jawed vertebrates (e.g. batoids, sturgeons and turtles). The paired basilars in both hagfish and lampreys give off mesencephalic and rhombencephalic transverse arterial branches (Fig. 4C). Hagfish brains show a dense anastomotic arterial network [37] that contrasts with a sparse central vasculature in lampreys which is formed mainly by isolated arteriovenous loops [39].

\section{Jawed Fishes (Gnathostomata)}

Gnathostomes consist of five subclasses: two extinct, Acanthodia and Placodermi, and three living, Chondrichthyes, Actinopterygia and Sarcopterygia [34]. Relationships of these five groups remain somewhat contentious, but what is generally agreed is that Cephalaspids are their nearest relatives among agnathans, and that ray-finned and lobefinned fishes comprise a group that excludes chondrichthyes, namely, the bony, jawed fishes, or Osteognathostomes.
Well-preserved fossil braincases are known for many early gnathostome taxa, in some cases allowing for speculative reconstruction of major cranial vascular pathways. Unfortunately, the fossil data does not preserve evidence of the cerebral vasculature.

\section{General Brainstem Vascular Design in Chondrichthyes}

Living chondrichthyans comprise sharks (selachians), batoids (skates, rays \& allies) and ratfish (holocephalans), the latter generally being considered as the sister-group of all other extant chondrichthyes [34]. With the exception of certain large lamnids that are specialized for cranial endothermy [41], the supply to cerebral vessels is generally similar throughout modern sharks [17, 42-46]. In most species, the internal carotid arteries (ICA) arise from the efferent hyoid arteries, often with contributions from anterior extensions of the paired dorsal aorta (Fig. 5A). After giving off the orbital arteries, the left and right ICAs turn medially in the roof of the mouth and meet below the base of the skull (Fig. 5A) to either form an arc-like commissural vessel (within the skull in Chlamydoselachus [43]) or cross each other at the midline, often with fusion of the vessels at the crossing point [47]. In either type, a short, unpaired ICA extends dorsally from the middle of this arc/crossing point and penetrates the thick cartilaginous posterior wall of the hypophyseal fossa [6, 17]. In Squalus, Scylliorhinus, Mustelus and other species, the unpaired ICA divides into paired cerebral carotids (CCA) that proceed laterally and rostrally against the interior walls of the hypophyseal fossa, ventral to CN III, where they are joined by the often larger
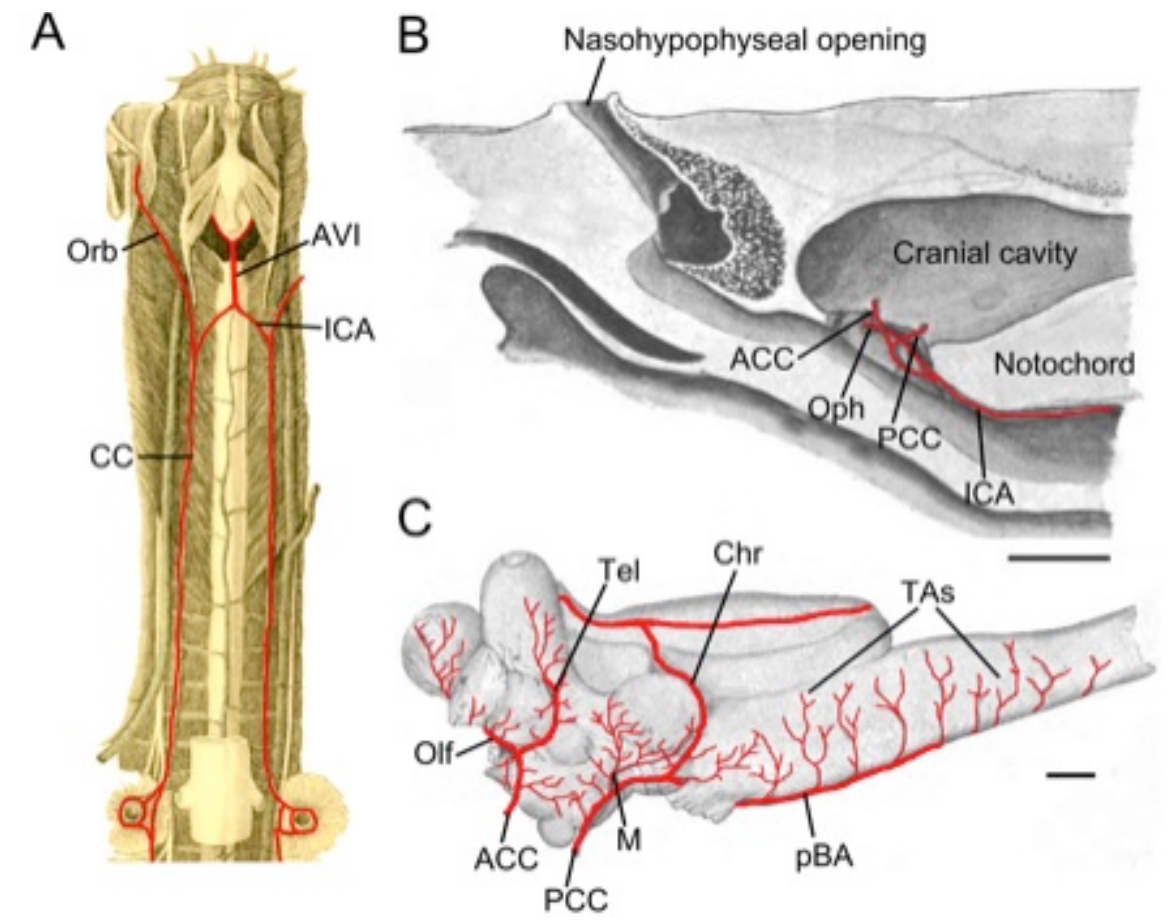

Fig. (4). Cyclostomes. A. Illustration modified after Müller [7] demonstrating the common carotid arteries (CC) traveling from the branchial region to the cranium and then dividing into orbital (Orb) and internal carotid (ICA) arteries. The ICAs join in the midline to form an arteria vertebralis impar (AVI). B, C. Illustrations modified after Sterzi [39] showing the cerebral vessels of the adult lamprey. B. The internal (cerebral) carotid artery (ICA) gives off an ophthalmic (Oph) branch before dividing into anterior (ACCs) and posterior (PCCs) cerebral carotids that enter the braincase separately, just rostral and caudal to the hypophysis. C. The ACCs give off olfactory arteries (Olfs) as well as telencephalic arteries (Tels) to supply the forebrain. The PCCs give off mesencephalic (M) and choroidal (Chr) arteries before continuing caudally as paired basilar arteries (pBA) that give off transverse branches (TAs). Scale bars are $5 \mathrm{~mm}$ in B and $1 \mathrm{~mm}$ in C. 
efferent pseudobranchial arteries (Fig. 5A, B). Near the optic nerves, the cerebral carotids divide into anterior (ACCs) and posterior (PCCs) cerebral carotid arteries (*in Fig. 5B). The ACCs run rostrally in the groove between the telencephalic lobes and give off large dorsal branches to the latter before continuing to the olfactory bulbs. The PCCs run caudally between the lateral walls of the brainstem and the hypothalamus, give off large diencephalic, mesencephalic and cerebellar branches and fuse in the midline between the oculomotor and trigeminal nerve roots to form an unpaired basilar artery (Fig. 5B; [22]). The basilar artery gives off a series of transverse rhombencephalic branches to each side of the hindbrain, in a quasi-segmental pattern [22]. Narrow penetrating branches arise from the basilar and transverse arteries [45]. Although the basilar artery in most sharks remains an unpaired midline vessel that is continuous caudally with the ventral spinal artery, paired basilars (Fig. 5C) and a number of cases of fenestrated or partially paired basilar arteries have been described in some sharks (Fig. 5E, F; $[42,48])$.

Most of the intradural parts of the cerebral vasculature in holocephalans (Fig. 5D), including the ACCs, PCCs, basilars and transverse branches, are similar in gross morphology to those just described in sharks [49]. The overall similarity between the cerebral carotid/basilar artery patterns of sharks and holocephalans suggests that their common features are primitive for Chondrichthyes as a whole. However, the origin of the carotid circulation from the efferent branchial vessels differs between these groups. Allis [50] showed that the brains in adult ratfish (Hydrolagus colliei) do not receive blood from internal carotids, but rather through "anterior" carotid arteries (Fig. 5G [50]), which are mandibular arch vessels that appear to be homologous to portions of the efferent pseudobranchial arteries of sharks (holocephalans lack pseudobranchs). The vessels in holocephalans that correspond to the proximal ICAs in sharks simply form a commissural arch linking the paired dorsal aorta. Allis [50] proposed that the more distal portions of the ICA in holocephalans regress during ontogeny (dashed lines in Fig. 5G). While the early stages of ICA formation have not been described, De Beer and Moy-Thomas [51] reported that internal carotid stems extending rostrally from the paired dorsal aorta were present in $95 \mathrm{~mm}$ embryos of Callorhynchus, but that the vessels did not enter the braincase.

The comparison between sharks and batoids is roughly converse to that between sharks and holocephalans. Batoids have a set of connections between the ICA and efferent branchial vessels similar to those in sharks, but their basilar arteries and branches differ considerably. As in sharks, the cerebral carotids in most batoids receive contributions from both the efferent hyoid and pseudobranchial arteries [9]. In most cases, there is either 1) an unpaired ICA as in sharks, seen in electric rays, 2) a transverse intercarotid commissure, seen in myliobatid rays, or 3) a simple crossing of the paired ICAs as they enter the skull, seen in skates [46]. In the skate Raja asterias (punctatus), the paired ICAs cross each other at the midventral line of the skull, and enter the hypophyseal fossa as paired CCAs [22]. The cerebral carotids are joined by the efferent pseudobranchial arteries and then divide into
ACCs and PCCs just caudal to the optic nerves. The PCCs run caudally along the sides of the midbrain and rostral hindbrain giving off ventral branches to the hypophyseal region as well as dorsal branches to the diencephalon, mesencephalon and cerebellum, in a pattern similar to sharks.

The remaining part of the "basilar" system in most batoids is unlike that in adult sharks, but similar to the general gnathostome embryonic pattern (Fig. 5C), in that the PCCs do not fuse to form an unpaired basilar artery, but continue to the spinal cord as paired basilars $[13,52]$. The paired basilars in skates send transverse vessels both medially and laterally into the hindbrain before merging into an extensive ventral arterial plexus that extends through the rostral spinal region (Fig. 5C). The diameter of the paired basilars increases spinalward, leading Grodzinski [22] to suggest a caudal origin of blood flow to the medulla through a 'vertebral' arterial supply.

Although fenestrated or partially duplicated basilar arteries have been described in sharks (Fig. 5E, F), most sharks and holocephalans have a median, unpaired basilar artery that runs the length of the hindbrain sending out transverse branches to both sides of the neuroepithelium. The lack of a pseudobranch and atrophy of the internal carotid connection in Holocephalans appears to have had no influence on the basic branching patterns of the PCC/BA. In contrast, batoids generally have paired PCCs that never fuse to form a basilar. While this could be interpreted as retention of the generalized developmental pattern for hindbrain vessels, skates and rays do not have other obvious features suggestive of pedomorphosis (retention of juvenile traits into adulthood). The presence of unpaired basilars in squatinaformes [46], a group often considered to be a basal member of the batoids, supports the likelihood that paired BA arose within batoids as a modification of the unpaired shark-ratfish pattern rather than as retention of a primitive gnathostome condition. As in most vertebrates, the main brain arteries in Chondrichthyes encircle the brain and send penetrating branches into the tissue, forming extensive anastomotic networks. The small size and capillary-like appearance of the penetrating vessels reported by Sterzi [53] may be unique to cartilaginous fishes.

\section{Ray-Finned Fishes (Actinopterygia)}

Actinopterygii are the largest group of extant vertebrate species and are diversified over time through a series of large scale radiations that have produced morphological, behavioral and ecological adaptations allowing them to occupy almost every aquatic habitat on Earth [54]. While almost all living actinopts are teleosts, a few basal groups, the polypterids, acipensers (sturgeons and paddlefish), bowfins and gars, provide a sample of the much broader diversity evident in the extensive fossil record of extinct actinopts, and have therefore been subjects of numerous classic anatomical and developmental studies $[55,56]$. The abundance and quality of actinopterygian fossils have made it possible to document evolutionary transformations of numerous neurocranial features that could not have been inferred from studies of only extant species [34]. These features include the paths of the internal carotid arteries into the cranial cavity through newly formed parabasal and 

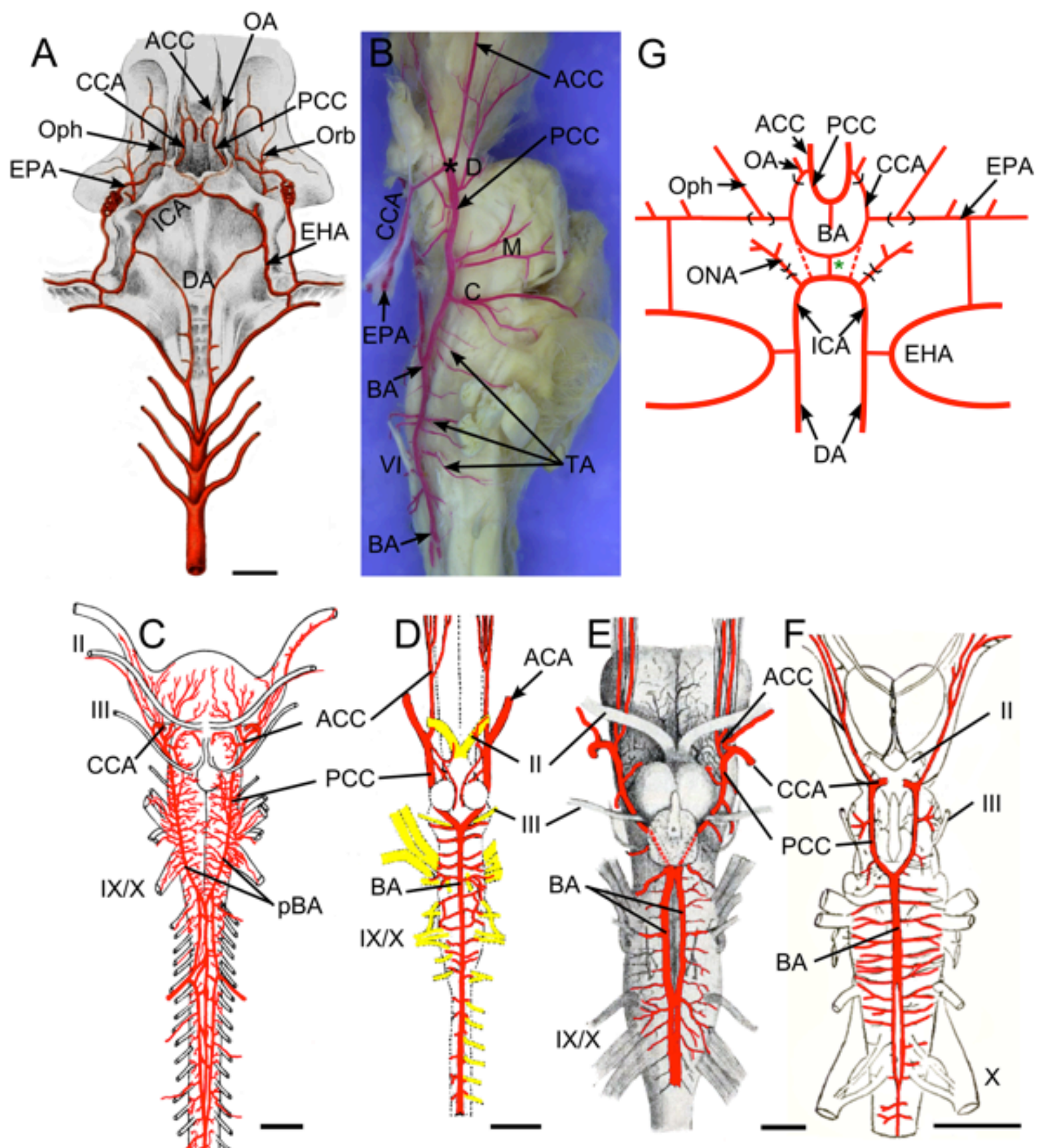

Fig. (5). Brain vasculature in Chondrichthyes . A. Arteries of spiny dogfish (Squalus acanthias) modified from Hyrtl [8] show paired dorsal aortae (DA) joining the efferent hyoid arteries (EHA) which divide into internal carotid (ICA) and orbital (Orb) arteries. Paired ICA fuse to form an unpaired carotid trunk that enters the braincase and divides into left and right cerebral carotids (CCA). Efferent pseudobranchial arteries (EPA) give off ophthalmic arteries (Oph) before joining the CCA in the braincase. The CCA bifurcate into anterior (ACC) and posterior (PCC) cerebral carotids. Optic arteries (OA) arise near that bifurcation. B. Lateral view of adult $S$. acanthias brain with red vascular fill showing the CCA dividing (*) into ACC and PCC. The PCC give off diencephalic (D), mesencephalic (M) and cerebellar (C) branches before fusing to form a midline basilar artery (BA) that gives off numerous transverse arteries (TA). Brain vessels of C, Raja clavata (thornback ray) after Hofmann [13]; D, Hydrolagus colliei (ratfish) after Craigie [49]; E, Cetorhinus maximus (basking shark) after Carazzi [42] and F, Squalus suckleyi (spotted spiny dogfish) after Daniel [48] show BA variations in different Chondrichthyes. As in most sharks, holocephalans (D) have an unpaired BA, while skates (C) and most other batoid fish have paired basilars (pBA). Partial duplications of the BA are shown in two elasmobranchs $(\mathbf{E}, \mathbf{F})$, likely representing variations within species that typically have a single BA. G. Schematic of vessels in ratfish after Allis [9]. The ICA extend rostrally from the DA and give off orbitonasal arteries (ONA) before joining in the midline. The unpaired carotid trunk (green ${ }^{*}$ ) and proximal part of the CCA (red dotted lines) are present in embryos and regress in adults. The main cerebral blood supply comes from "anterior carotid" arteries (homologous to EPA of elasmobranchs) that give off ophthalmic arteries (Oph) before entering the brain case as CCA and dividing into ACC and PCC. The PCC fuse to form an unpaired BA. II, optic nerve; III, oculomotor nerve; IX, glossopharyngeal nerve; X, vagus nerve. Scale bars are $1 \mathrm{~cm}$ in $\mathbf{A}, \mathbf{C}, \mathbf{E}, \mathbf{F}$ and $2.5 \mathrm{~mm}$ in $\mathbf{D}$. 
carotid canals, the formation of the posterior myodome, and the locations of cranial nerve roots and cerebral veins traversing the skull $[57,58]$. The posterior myodome, or eyemuscle canal, is a conical space located at the base of the cranium, found only in Amia and teleosts that houses some of the rectus muscles of the eye $[16,57,58]$. In most teleosts, the posterior myodome lies between the parasphenoid bone and the braincase proper. The internal carotid arteries generally enter this space and fuse to form an unpaired carotid that passes dorsally between the myodomes to enter the hypophyseal fossa.

\section{Basal Actinopterygians}

\section{Cladistia}

The Cladistia (Polypteriformes), comprising bichirs, reedfish and ropefish is a relict group lying at the base of the actinopterygian radiation. Classic studies by Allis [56] and others have documented the cranial anatomy of Polypterus in great detail, including the basic pattern of non-cerebral cranial vasculature. There have not been, however, any studies of cerebral vasculature in these fish. For the present study, the thick cartilaginous cranial base underlying the hypophyseal fossa and hindbrain was dissected in uninjected specimens of Calamoichthyes $s p$ to display the ventral cerebral vessels. Although the material was poorly suited for detailed dissection and photography, paired basilars could be visualized with a dissecting microscope because of a covering of pigment cells on the otherwise nearly transparent vessels. While not surprising, this result needs to be verified with studies based on vascular fills. As will be seen in the subsequent groups, the presence of paired basilar arteries appears to correlate with a highly cartilaginous adult neurocranium.

\section{Chondrostei}

The only extant Chondrostei are the Acipenseriformes, which include sturgeons and paddlefish. The branchial arch vessels and basic arterial supply to the brain have been described in the American paddlefish, Polyodon spathula, by Danforth [59] and in sturgeons by Demme [60] and Grodzinski [61]. However, the vascular patterns in these groups are similar, some differences do exist. Un-injected specimens of paddlefish with alcian and alizarin counterstain were dissected for comparison with Danforth's results.

The carotid arteries in the American paddlefish (Polyodon spathula) and the sterlet (Acipenser ruthenus) have similar relations and branches, despite apparent differences in the accounts of Danforth [59] and Grodzinski [61]. The carotids arise from the first efferent branchial arteries and divide into large orbital arteries (ext. carotid of Danforth) and diminutive internal carotid arteries [59]. Grodzinski misinterpreted the orbital arteries as "efferent hyoid arteries" [61]. The ICAs run anteriorly in the roof of the mouth on the ventrolateral surface of the parasphenoid bone and after entering the 'carotid canal' get joined by the larger efferent pseudobranchial arteries [59]. After this anastomosis, the efferent pseudobranchial arteries become reduced and continue as the ophthalmic arteries, while the ICAs (now CCAs) become more robust and penetrate the sides of the basal cartilage to enter the braincase lateral to the hypophysis. Grodzinski did not describe connections between the efferent pseudobranchial arteries and the internal carotids. Such connections, similar to those observed by Danforth [59] in paddlefish, were however described by Demme [60] in adult sturgeon [61]. Holmgren [62] reported embryonic anastomoses of the internal carotid and efferent pseudobranchial arteries and it is possible that these connections become reduced during further development in some sturgeons.

The remaining cerebral vessels are similar in sturgeons and paddlefish. The CCAs in both groups divide into ACCs and PCCs lateral to the optic chiasm. The PCCs in the sterlet pass below the root of $\mathrm{CN}$ III and fuse together to form a median trunk running between the saccus vasculosus and the ventral surface of the midbrain (Fig. 6A). The trunk branches into paired basilar arteries that give off numerous long and short transverse branches to supply the midbrain, cerebellum, and hindbrain. The PCCs in the paddlefish do not form a single median trunk as in sturgeons, but simply have a short commissural vessel joining the paired PCC as they become paired basilars. The transverse rhombencephalic arteries in both groups include branches that supply the trigeminal, vestibulocochlear glossopharyngeal and vagus nerves.

The carotid arteries in acipenseriformes show many conserved anatomical features similar to chondrichthyes along with a few unique characters. In sturgeons and paddlefish, the branching of paired lateral aorta, orbital, efferent pseudobranchial and ophthalmic arteries with respect to the origin of the cerebral carotids is very similar to that seen in sharks. The major difference is that the ICAs/CCAs in Chondrostei remain paired, and widely separated as they enter the hypophyseal fossa; no unpaired carotid segment as seen in sharks or in Teleosts (below) is present. Whether this is a primitive feature similar to the condition in lampreys, or the result of modification of an elasmobranch-like pattern is unknown.

\section{Amia and Gar}

Despite the large number of anatomical studies of the cranial anatomy of bowfins and gars, including details of the braincase and the pseudobranchial circulation, very little is known about the brainstem vasculature [5, 6, 16]. Allis described the basic branches of the carotid system in adult bowfin [5], while Shearer [63] reconstructed cranial vessels in embryonic stages (Fig. 6B). Those results are compared here with dissections of un-injected adult gar (Lepisosteus osseus) and both adult and larval bowfin (Amia calva). Anterior to the union of the first two aortic arches, the paired dorsal aorta continue forward as the internal carotid arteries in both gar (Fig. 6C) and bowfin (Fig. 6B; [63]). In gar, the ICAs pass along the medial side of the hyomandibular cleft before entering the carotid canal where they are joined by the equally large efferent pseudobranchial arteries to form CCAs. The CCAs give off ophthalmic arteries before dividing into anterior and posterior cerebral carotid arteries. The choroid rete is not present in gars, but the ocular choroid is still supplied by an ophthalmic artery. In Amia, the connection between the cerebral carotid and EPA is not as robust as in gar, with Allis describing merely a small caliber branch joining them [5]. These differences are thus nearly identical to the differences between paddlefish and sturgeon. The ophthalmic artery (OA) in embryonic Amia was shown 

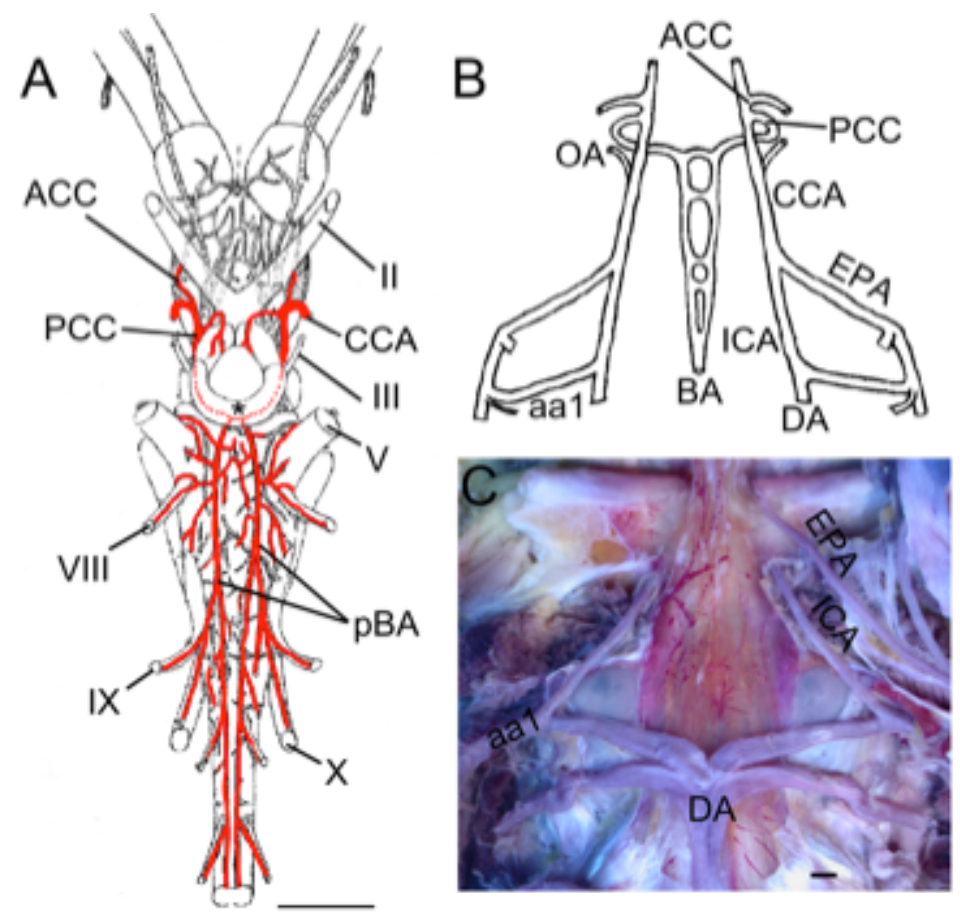

Fig. (6). Basal Actinopts. A. Illustration modified from Grodzinski [61] showing the brain vessels of Acipenser ruthenus. The cerebral carotids (CCAs) divide into anterior (ACCs) and posterior (PCCs) divisions. The PCCs fuse to form a common crossbridge (black *) and continue as paired basilars (pBAs). Rhombencephalic transverse branches arise from both basilars and include vessels that supply $\mathrm{CN}$, VIII, IX, and X. B. Schematic reconstruction of larval Amia calva modified after Shearer [63] showing the internal carotids (ICAs) as rostral extensions of the dorsal aorta (DA). The efferent pseudobranchial arteries (EPAs) join the ICAs outside of the braincase. The ICAs continue as CCAs that give off ophthalmic arteries (OAs) before dividing into ACCs and PCCs. The PCCs in embryos and larva continue caudally as paired basilar arteries with multiple cross bridges between them (BAs). C. Dissection of adult gar (Lepisosteus osseus) showing the paired ICAs arising from the dorsal aorta (DA) and first epibranchial artery (aa1). The EPAs enter the braincase in close proximity to the ICAs. II, optic nerve; III, oculomotor nerve; V, trigeminal nerve; VIII, vestibulocochlear nerve; IX glossopharyngeal nerve; X, vagus nerve. Scale bars in $\mathrm{A}, \mathrm{C}$ are $2 \mathrm{~mm}$.

by Shearer [63] to originate as a branch of the ICAs (Fig. 6B), while in adults it is a vessel running from the pseudobranch to the choroid rete mirabile of the eye with no or only a small connection to the main carotid line. Branches of the CCA in Amia are similar to other vertebrates, with an ACC division supplying the forebrain, while the PCCs in embryos and larva (Fig. 6B) continue caudally as paired basilar arteries with multiple cross bridges between them [63]. The paired basilars in large adult Amia may fuse together to form an unpaired basilar, since Allis [5] described the PCCs as forming a circulus cephalicus with a median extension running forward into the midbrain and another running caudally beneath the midline of the hindbrain.

\section{General Cerebral Vascular Pattern in Basal Actinopterygians}

Some features of the carotid arterial pathways are similar between basal actinopterygians and chondrichthyes. Similar anatomical relationships between paired dorsal aortae, efferent hyoid and pseudobranchial arteries and the origins of orbital, ophthalmic and optic arteries are seen across these groups. Basal actinopts lack the unpaired carotid trunk or commissure between the origins of the orbital and ophthalmic arteries that are seen throughout chondrichthyes. The presence of paired basilar arteries in Cladistia, acipenserformes and bowfins/gars suggests that this condition is primitive for ray-finned fishes.

\section{Teleosts}

Teleosts are the most diverse and species-rich group of vertebrates. Although some aspects of adult brain vasculature have been described for a few species, none have been examined in any detail and most of the larger taxonomic groups have no descriptions at all. The present review will therefore be limited to a few species, sampled from across the Teleostei, with the intention of gaining an initial view of the distribution of known anatomical characteristics across the group as a whole (Fig. 7A). Of the species considered, the Ostariophysi are represented by the cyprinids, goldfish (Carassius auratus) and zebrafish (Danio rerio), the Salmoniformes by the rainbow trout (Salmo irideus) and the Percomorpha by two distantly related members of this huge group, the lingcod (Ophiodon elongatus) and white perch (Morone americana). The overall vascular supply of the adult brain has been previously described for the lingcod [21] and the rainbow trout [10] (Fig. 7B). The development of brain vessels has been examined in rainbow trout [64] and zebrafish [31-33]. Original work on the central arteries of goldfish and zebrafish as well as on the carotid supply in white perch extends previous studies and allows a better estimate of the general pattern across teleosts. 


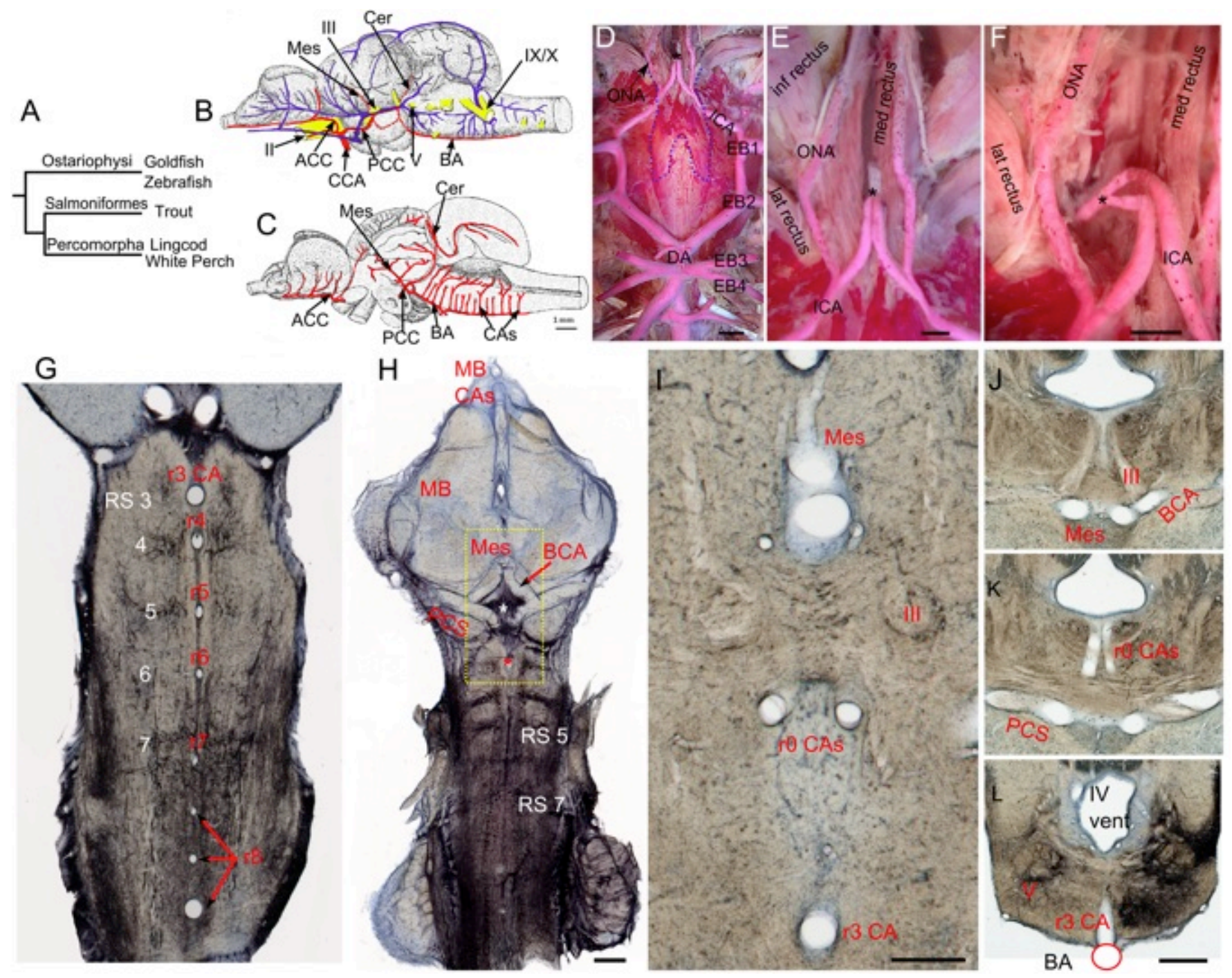

Fig. (7). Teleostean hindbrain vasculature. A. Taxonomic tree showing relationships among the teleost fish examined. B-C. Trout brain vessels in lateral (B) and medial (C) views modified after Grodzinski [10]. The cerebral carotids (CCA) divide into anterior (ACC) and posterior (PCC) divisions, with the paired PCC giving off mesencephalic (Mes) and cerebellar (Cer) branches before fusing to form an unpaired basilar artery (BA). The BA gives off multiple midline rhombencephalic penetrating branches, termed central arteries (CAs). D-F. Adult white perch (Morone americana) with red latex vascular fill showing the internal carotid arteries (ICA) extending rostrally from the dorsal aortae (DA), giving off orbitonasal branches (ONA) and fusing to form an unpaired carotid arterial trunk (black *). The border of the posterior part of the removed parasphenoid bone is shown by the blue dashed line. G. The segmental pattern of midline CA stems in rhombomeres (r) 3 to $\mathrm{r} 8$ are shown in a horizontal section of an adult goldfish hindbrain retrogradely labeled with biocytin and counterstained with cresyl violet. The reticulospinal neurons (RS) are located at the center of each rhombomere on either side of the CA. $\mathbf{H}$. Projection of 5 horizontal sections of the same specimen showing RS neurons and the $\mathrm{r} 3 \mathrm{CA}$ (red *). The caudal divisions of the ICA send basal communicating arteries (BCA), that fuse in the midline and give off mesencephalic arteries (Mes) and posterior communicating segments (PCS) that give off r0 CAs (white *) before fusing together to form an unpaired BA. I. High magnification view of the yellow box in $\mathbf{H}$ showing the Mes, r0 CAs, and r3 CA as clear tubes. The oculomotor nerve (III) lies between the Mes and r0 CAs. J-L. Transverse sections of another goldfish specimen show the oculomotor nerve path $(\mathbf{J})$, the r0 CAs coming off the PCS (K), and the r3 CA arising from the BA (L). II, optic nerve; V, trigeminal motor nucleus; IX, glossopharyngeal nerve; X, vagus nerve; inf, lat and med rectus = inferior, lateral and medial rectus muscles. Scale bars are $2 \mathrm{~mm}$ in $\mathbf{D} ; 1 \mathrm{~mm}$ in $\mathrm{E}$ and $\mathbf{F} ; 1 \mathrm{~mm}$ in $\mathbf{H} ; 250$ micrometers in $\mathbf{I} ; 1 \mathrm{~mm}$ in $\mathbf{J}-\mathbf{L}$.

\section{Connections of Internal Carotid Arteries in Teleosts}

The primary brain vascular supply in these species comes from paired internal carotid arteries (ICA) that extend rostrally from the paired dorsal aorta (Fig. 7D) $[5,10,21]$. The ICAs enter carotid canals which lie between the parasphenoid and basioccipital bones. Orbital arteries that supply some of the extraocular muscles and extend rostrally through the palatal region to the nasal cavities arise from this portion of the ICAs. The presence and location of intercarotid commissures or of fully unpaired ICA segments (encephalic) differ among teleosts. In perch (Fig. 7D-F), trout [10], and tilefish [21], the left and right ICAs join to form a median unpaired carotid trunk, that passes dorsally between the medial rectus muscles in the posterior myodome before entering the braincase near the hypophysis through a median opening in the basisphenoid (asterisk in Fig. 7D-F). 
The unpaired carotid pierces the hypophyseal fossa and branches into paired cerebral carotid arteries (CCA). An unpaired carotid does not appear to be present in larval zebrafish [31], and in sections of adult goldfish, a carotid commissure was present in the hypophyseal fossa, but there was no sign of a midline arterial trunk of the kind seen in the perch (data not shown). Similarly, among other cypriniformes, an unpaired carotid segment appears to be missing while a carotid commissure may be present (Nemachilus) or absent (Phoxinus) [65]. The eel Anguilla anguilla, a more basal teleost than the cypriniformes, likewise lacks an unpaired carotid segment [65], suggesting that the median trunk seen in perch may characterize more derived teleosts such as percomorpha. Efferent pseudobranchial arteries, that carry hyperoxygenated blood from the pseudobranch, travel cranially and medially, and enter the braincase where they either join the cerebral carotids (e.g. Salmo) and then branch off as ophthalmic arteries, or, as in most teleosts, remain separated from the carotids and simply become the ophthalmic arteries to supply the specialized choroid rete mirabile of the eye [6, 62], which is found only in Amia and teleosts [66]. The anatomy and development of the proximal parts of the cerebral carotid and efferent pseudobranchial arteries and their relationships to the posterior myodome have been described in a number of other species (Esox, Salmo, Gadus, etc.) by Allis [5], de Beer [55], Holmgren [62] and others. These structures will not be reviewed here since they are highly variable among teleosts and do not appear to correlate with clear differences in brainstem vascular anatomy.

\section{Carotid/Basilar Branches in Teleosts}

Regardless of midline connections, the cerebral carotids in all the species considered here enter the hypophyseal fossa just caudal to the optic nerves and immediately divide into anterior and posterior cerebral carotid arteries (Fig. 7B). The optic arteries, which travel with the optic nerves, arise near the bifurcation of the anterior and posterior divisions. The ACCs extend rostrally along the basal telencephalon and olfactory tracts, giving off branches that supply the forebrain and anterior parts of the midbrain. The PCCs (posterior communicating segments of Isogai et al. [31]) travel caudally from CN II, penetrate between the inferior lobe of the hypothalamus and the midbrain, and join to form the basilar artery (Fig. 7C) at the posterior aspect of the saccus vasculosus. On the way, the PCCs send medial branches that anastomose to form a large commissural vessel $(=$ basal communicating artery [31]) just rostral to the oculomotor nerves (Fig. 7H). The midline fusion that forms the basilar artery usually is found just rostral to the level of the trigeminal nerve root. The basilar continues caudally beneath the hindbrain and terminates by forking into transverse connections to the segmental spinal vessels near the hindbrain-spinal cord junction [21, 31]. In salmon, goldfish, zebrafish and midshipman, a series of large penetrating arteries arises from the basal communicating, posterior communicating and basilar arteries. These penetrating arteries are unlike those seen in other vertebrates in that they extend as large caliber vessels to the subventricular zone of the brainstem where they ramify extensively into elaborate intramural vascular trees. The basal communicating artery sends large dorsally directed central mesencephalic arteries into the floor of the midbrain just rostral to the oculomotor nuclei (Fig. 7I, J). These ramify throughout the ventricular region of the optic tectum and torus longitudinalis [10]. In goldfish, a smaller pair of penetrating arteries ( $\mathrm{r} 0$ central arteries) arises from the posterior communicating segments and enters the hindbrain caudal to the oculomotor nerve (Fig. 7I, K). The penetrating arteries arising from the basilar form a segmental series of midline vessels (hindbrain central arteries) that supply most of the hindbrain and much of the cerebellum. The complete series of hindbrain central arteries (CAs) have been demonstrated in trout (Fig. 7C; [10]), zebrafish [31], goldfish (Fig. 7G), loach and pike [65]. The CAs branching from the basilar are in concert with larval and adult rhombomeric neural structures such as segmental reticulospinal nuclei, beginning with $\mathrm{r} 3$ and extending up to r8. While Grodzinski [10] described an alternating series of larger and smaller hindbrain CAs in trout, the size differences seen within the series in cyprinids are much more pronounced. In goldfish and zebrafish, the vessels seen in $\mathrm{r} 3$ and in the rostral part of $r 8$ are significantly larger, both in stem diameter and in the degree of branching, than the other hindbrain vessels.

\section{Overall Teleost Hindbrain Vascular Architecture}

The presence of similar anatomical features in distantly related species such as cyprinids, salmonids and percomorphs demonstrates aspects of brain vasculature that are likely to be general for teleosts as a whole. Although teleosts conform to the general mature vertebrate pattern in which the posterior divisions (PCCs) of the cerebral carotids form the basilar artery, they are unique in possessing a segmental series of unpaired, midline hindbrain CAs extending from the basilar. Similar central arteries originate from the paired PCCs (= basal and posterior communicating segments) and penetrate the medial parts of the midbrain and rostral hindbrain. This overall pattern is retained throughout ontogeny and parallels the rhombomeric neuronal pattern [29]. Although midline or paramedian penetrating branches from the basilar are present in other vertebrates, they usually supply only a limited territory near the midline.

\section{Lobe-Finned Fishes (Sarcopterygia)}

Living Sarcopterygii, or lobe-finned fishes, include coelacanths, lungfish, and tetrapods. The two former groups are relicts of early successful sarcopt lineages and are famous as "living fossils", but neither can be viewed as being representatives of "primitive" sarcopts as they possess numerous derived features [67]. None of the living tetrapods are particularly rich in primitive sarcopt features either; hence the necessity for comparative and developmental studies to link features of extant groups with the extensive fossil record of extinct sarcopts. Detailed braincase anatomy is known for diverse extinct sarcopts, including close relatives of the earliest tetrapods [68]. The eye-muscle myodomes characteristic of actinopts are absent in sarcopts, while a fully formed intracranial joint seen in early sarcopts and living coelacanths is absent in actinopts [34]. An unpaired carotid trunk entering the hypophyseal fossa as is seen in most Chondrichthyes and Actinopterygia is not present in Sarcopterygia. Although the fossil materials illuminate sarcopt interrelationships and provide evidence 
for the paths of major carotid and venous channels, they do not reveal any features of the cerebral vasculature.

\section{Coelacanth (Actinistia)}

Coelacanths are the most basal extant sarcopterygians and include two known living species: Latimeria chalumnae and Latimeria menadoensis [34]. The gross morphology of coelacanth brains has been described in a number of studies $[69,70]$, but the brainstem vasculature is not well known. The internal carotid arteries arise as rostral extensions of the dorsal aorta, similar to other vertebrates, but do not run between the parasphenoid and basisphenoid as in other fishes, instead, they enter carotid foramina in the sphenoid cartilage to reach the hypophyseal fossa [71]. Although Lagios [72] mentioned an intercarotid commissure (as in lungfishes, see below), the branching of the CCAs into anterior (ACCs) and posterior (PCCs) cerebral carotids within the fossa and the further anatomy of the PCC branches supplying the hindbrain remain unclear. However, serial sections of the head of a coelacanth "pup", in the American Museum of Natural History in New York (I32949), show the presence of paired basilar arteries beneath both the rostral and caudal hindbrain. Transverse branches off of the paired basilars are also evident. Unfortunately, most of the sections containing the intermediate portions of the hindbrain are missing from the series and it is therefore not clear whether the basilars are paired throughout the length of the hindbrain or instead have an unpaired portion. Further studies are needed to determine the rostral and caudal connections of the paired basilars in Latimeria and the degree of conservation between these vessels and those in lungfish and basal actinopterygians.

\section{Lungfish (Dipnoi)}

In adults of the Australian lungfish Neoceratodus, studied by Spencer in 1893 [73] (Fig. 8), the cerebral carotid arteries are branches of so-called 'anterior carotid' arteries (ACAs) that originate from the efferent hyoid vessels. The ACAs penetrate the cartilaginous braincase at the posterior end of the skull [73]. Behind the infundibulum, they give off medial branches that unite to form an intercarotid commissure (Com), and hence a 'circulus cephalicus'. The main branches continue into the braincase as cerebral carotids (CCA) and divide into anterior and posterior cerebral carotid arteries [73]. Rostral extensions of the dorsal aorta, termed posterior carotids (PCA) by Spencer, pass near the origin of the cerebral carotids but do not connect with them (Fig. 8), instead giving off orbital arteries within the braincase. Kellicott [74], studying developmental series of Neoceratodus, showed that these relations are secondarily acquired during ontogeny and represent a capture of the cerebral carotids by growing extensions of the hyoidean efferent artery. The 'true' carotid stems thus originated as branches of the paired dorsal aorta as in other vertebrates [74]. The adult configuration is superficially similar to that in sharks and holocephalans where a vessel from the mandibular arch (efferent pseudobranchial in sharks, anterior carotid in ratfish) supplements or replaces the carotid supply from the lateral aorta. In Neoceratodus, however, there is no pseudobranch. It therefore appears that the newly formed "anterior carotid" of lungfish is analogous and not homologous to the "anterior carotid" of Holocephalans [49].
The branches of the cerebral carotids were not described in any detail by Spencer [73], Kellicott [74], or others. Nevertheless, it is clear from their figures that the ACCs travel rostrally, giving off olfactory and telencephalic branches to supply the forebrain, while the PCCs continue as paired basilar arteries that send transverse rhombencephalic branches to supply the brainstem (Fig. 8). Adult and

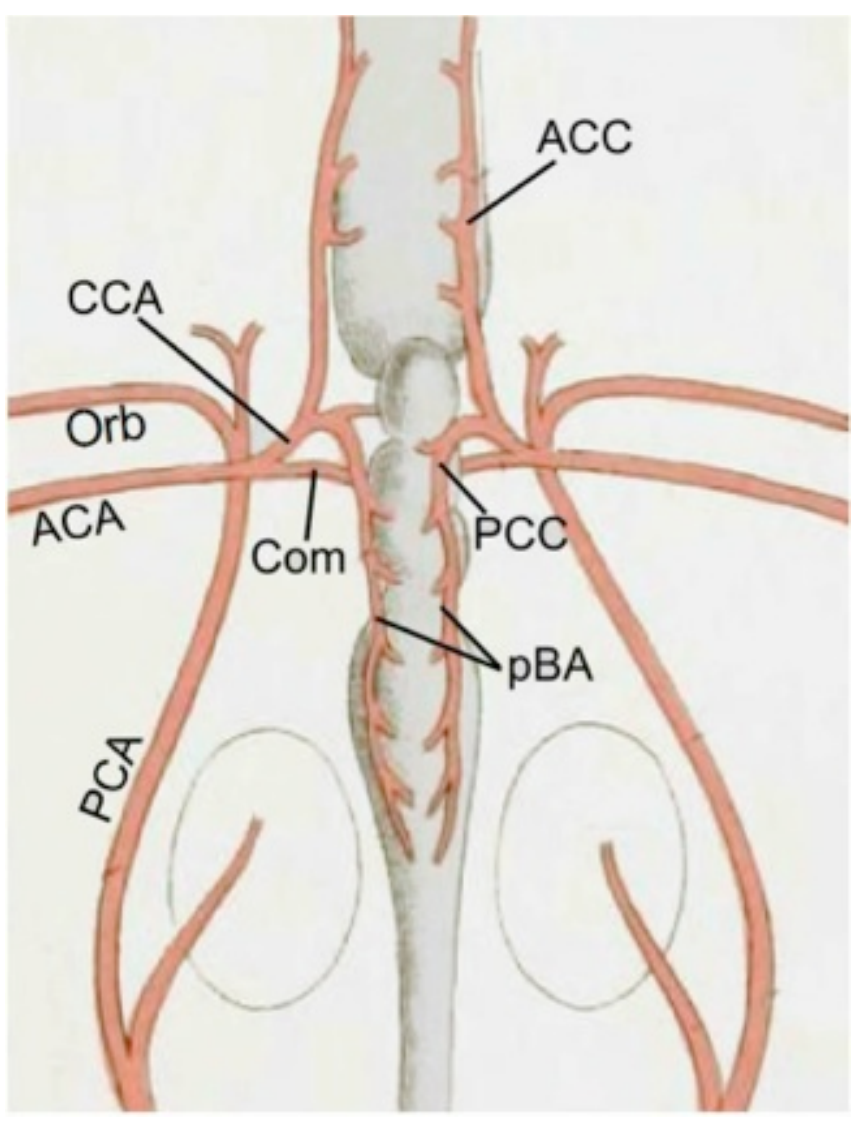

Fig. (8). Lungfish. Cerebral vasculature of Australian lungfish Neoceratodus modified after Spencer [73] shows the cerebral carotids (CCA) and an intercarotid commissure (Com) arising from anterior carotids (ACA). The CCAs divide into ACC and PCC divisions within the braincase. The PCCs continue as paired basilars (pBAs) that give off transverse branches. Posterior carotid arteries (PCAs), which represent remnants of the original ICA trunk, extend rostrally from the paired dorsal aorta and continue as the orbital arteries (Orbs) without connecting with the CCA.

developing cranial vasculatures of the South American lungfish, Lepidosiren, were briefly described by Robertson [75]. Although her description of cerebral arteries is incomplete and employs an idiosyncratic terminology, it appears that Lepidosiren has an intracranial carotid commissure and paired basilar arteries as in Neoceratodus. The likely paired basilar arteries supplying the hindbrain are termed "vertebro-cerebral arteries" and are said to arise posteriorly from the dorsal aorta near its junction with the efferent branchial vessels. The caudal ends of these vessels where they join the dorsal aorta would therefore correspond either to the "primitive hypoglossal arteries" or the first spinal segmental arteries seen in many taxa $[14,26,31,76]$. Further investigations within lungfish are needed to determine whether the basilar arteries in Neoceratodus are 
similarly connected with the dorsal aorta and to search for similarities and differences between the paired basilars of lungfish and those of batoids and acipenserids.

\section{Amphibians (Lissamphibia)}

Living amphibians are usually grouped together as the Lissamphibia, comprising salamanders, frogs and caecilians, and excluding most known fossil amphibian taxa. Because of their highly specialized body plans, none of the extant groups are considered to be good stand-ins for early amphibians. Numerous works have examined aspects of cardiovascular anatomy in salamanders and frogs without providing much detail about the overall cerebral vascular pattern. Microvascular studies have shown loop, network and intermediate brain vessel types in amphibians. While general anatomical descriptions are available for cerebral vessels in salamanders and frogs, such studies appear to be lacking for caecilians.

\section{Salamanders (Urodela)}

The most complete descriptions of endocranial blood vessels in Urodeles are those of Francis [24] for Salamandra salamandra (fire salamander) and Roofe [76] for Ambystoma tigrinum (tiger salamander), which are similar in most regards. The brain blood supply in urodeles comes from cerebral carotid branches similar to those of most fishes. The common carotid arteries emerge from the truncus arteriosus anterior to the systemic arch and divide into external and internal carotid branches near the carotid gland [24]. After giving off ophthalmic branches, the ICAs enter carotid canals between the parasphenoid bone and floor of the braincase. Passing forward in the canals, they enter the braincase through the basicranial fenestra as cerebral carotid arteries (CCAs), and immediately divide into anterior (ACCs) and posterior (PCCs) cerebral carotid arteries (Fig. 9A-C). In Ambystoma, each PCC turns caudally and gives off ventral branches to the pituitary region, a large dorsal mesencephalic branch to the tectum and one or more small commissural branches that unite in the midline rostral to the roots of CN III (Fig. 9A). The PCCs run caudally between the brainstem and hypothalamus, arcing towards the midline where they join to form the basilar artery. The junction lies beneath the rostral hindbrain, in front of the trigeminal nerve roots. The basilar artery gives off numerous transverse vessels including large trigeminal (Va, Fig. 9A, B) and auditory branches before becoming continuous with the ventral spinal artery at the level of the first spinal artery [76]. Overall, the transverse arteries in both Salamandra and Ambystoma form a symmetric, ladder-like series of vessels supplying the medullary and pontine regions, although exact numbers, segmental relations and territories have not been described. Roofe [76] noted variations in Ambystoma where the basilar artery appeared as two paired vessels with multiple anastomotic bridges connecting them. The main difference between Salamandra and Ambystoma is that the PCCs in the former turn medially and unite in the midline rostral to the roots of CN III, forming a cross-bridge from which parallel caudal branches emerge that fuse into a single unpaired basilar artery (Fig. 9B). Other species of Salamandra have PCCs and commissural connections that are more similar to Ambystoma (Fig. 9C). Craigie [1] reported that most salamanders have simple loop-type cerebral microvasculature, while some larger-sized genera such as Ambystoma have the loops interspersed with regions supplied by simple anastomotic networks. Tsuneki and Ouiji [77] showed that intraparenchymal brain vessels are almost completely lacking in numerous species of the primitive urodele family Hynobiidae. These species are sluggish animals living in very cold habitats and have extensive choroid plexuses that presumably supply nutrients to the CNS through the cerebrospinal fluid. Whether they also lack the main channels of the carotid-basilar system does not seem to have been reported.

\section{Frogs (Anura)}

Dissection of several vascular-injected bull frogs (Rana catesbiana) confirmed the classical descriptions of Ecker and Gaupp [11]. The common carotid arteries arise from carotid arches, give off palatine (= orbital) arteries, cross the parasphenoid and ascend into the orbit where they divide into internal carotid and ophthalmic arteries [11]. The ICAs travel cranially and medially before piercing the hypophyseal fossa (now becoming the cerebral carotid arteries) and dividing near the optic nerve into anterior and posterior cerebral carotid arteries. The PCCs run caudally and medially, giving off mesencephalic and pituitary vessels, before fusing together to form a short crossbridge at the level of the oculomotor nerve roots (Fig. 9D). Paired basilars extend caudally from the crossbridge before merging in the midline to form an unpaired basilar artery that gives off transverse branches to both sides. Although all bull frogs examined in this study $(\mathrm{N}=6)$ had a midline unpaired $\mathrm{BA}$, they varied in the length of the initial paired portions of the basilar, depending on where the fusion of the PCCs occurred (Fig. 9E, F). The CNS microvasculature in adult anurans forms an anastomotic network both in the brain and spinal cord [28].

The pathways and branches of the CCAs in frogs and salamanders, such as neural territories supplied by particular branches show strong similarities not only within amphibians, but in comparison to generalized bony fishes. The large-caliber paired PCCs in both salamanders and frogs are connected across the midline by a commissural vessel, similar in topography to, but almost certainly not homologous with, the basal communicating segment of the PCCs in teleosts. In salamanders, a midline unpaired basilar is either formed from the fusion of the main PCC vessel with its counterpart or from the fusion of caudally-directed branches from the arc-like PCC commissure. Similar to salamanders, frogs have parallel branches arising from the commissure that travel caudally for a certain length before fusing to form an unpaired basilar. It seems possible that more highly pedomorphic salamanders such as Proteidae may show even greater tendencies to retain paired basilar arteries in adults.

\section{Amniotes}

Amniotes contain a wide range of taxa with cerebral blood flow that differs in direction and source. Regardless of whether blood travels in a rostral to caudal direction through the carotid-basilar arterial system (i.e. turtles, lizards, birds) or from caudal to rostral through a vertebro-basilar system (mammals), the basilar artery remains the main blood supply of the hindbrain. Developmental modifications have created 

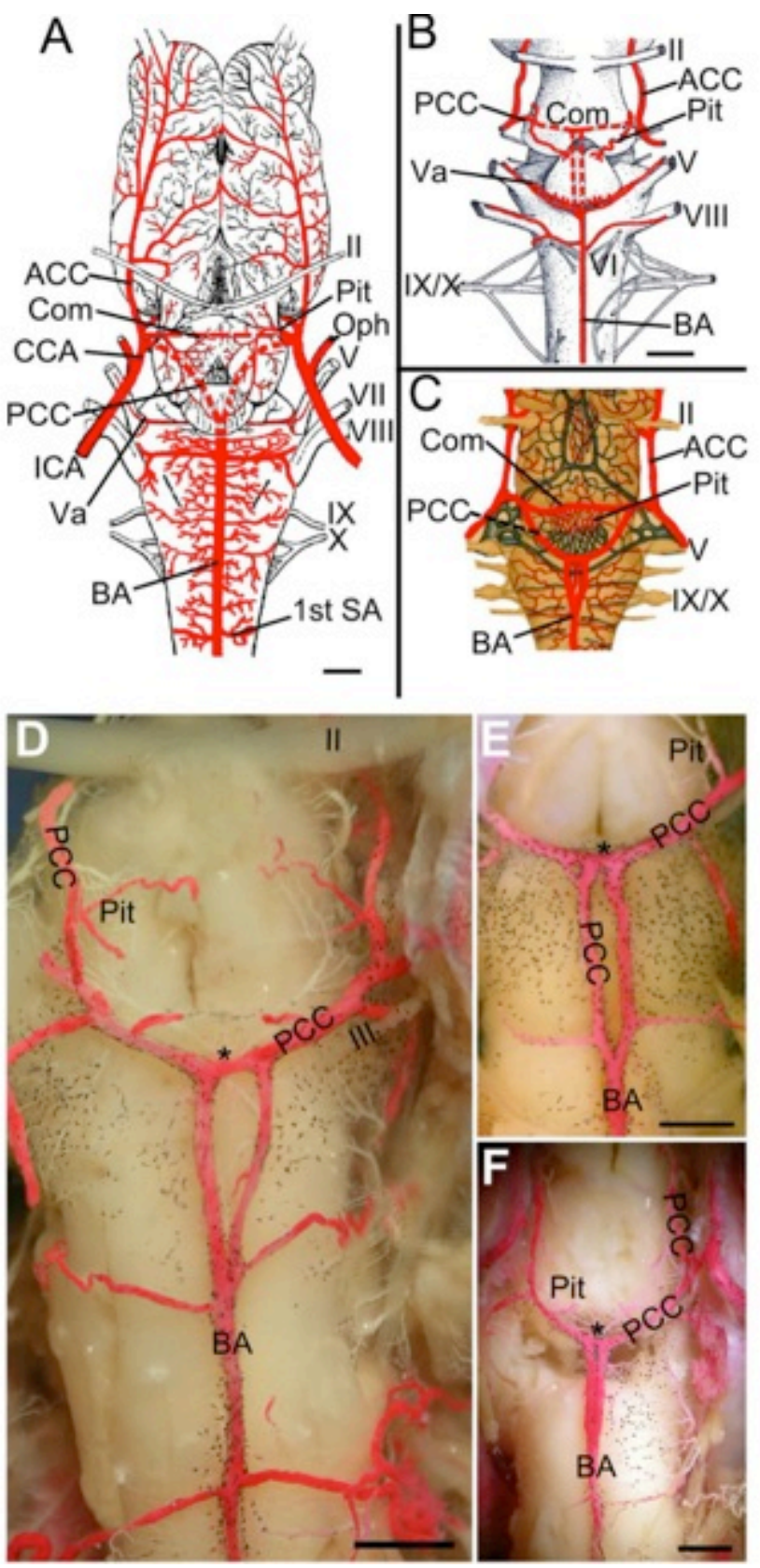

Fig. (9). Amphibians (Salamander and Frog). A. Cerebral vasculature of Ambystoma tigrinum modified after Roofe [76] showing the cerebral carotid arteries (CCAs) giving off ophthalmic arteries (Ophs) and dividing into anterior (ACC) and posterior (PCC) divisions. The PCCs give off pituitary branches (Pit), form a short crossbridge (Com), and then join (red dotted line) to form a midline unpaired basilar artery (BA) that gives off numerous transverse branches, including large vessels traveling with trigeminal (Va) and vestibulocochlear nerves. B. Illustration of Salamandra salamandra modified after Francis [24] showing the unification of the PCCs to form a crossbridge (Com; red dotted lines) and giving off two parallel caudal branches (red dotted lines) that join to form an unpaired BA. Pituitary (Pit), trigeminal (Va) and vestibulocochlear branches are also shown. C. Illustration of Salamandra maculosa modified after Schöbl [23] showing a third variation of the unpaired BA formation that combines features seen in A and B. No magnification was given by Schöbl for C. D-F. In Rana catesbiana, although the PCC travel caudally and fuse to form the BA, the length they travel paired varies in different specimens. The PCC give off pituitary and mesencephalic branches, form a short crossbridge $(*)$, and then travel caudally before fusing to form an unpaired BA. All specimens had symmetrical transverse branches coming off the BA. A partially fused BA with a paired region (fenestration) just caudal to the PCC crossbridge (F) was also found. II, optic nerve; V, trigeminal nerve; VII, facial nerve; VIII, vestibulocochlear nerve; IX, glossopharyngeal nerve; $\mathrm{X}$, vagus nerve. Scale bars are $1 \mathrm{~mm}$ in A-B and $1 \mathrm{~mm}$ in D-F.

an array of variations within amniotes, including the differing branching patterns of ACC-PCC, which can change the origin of main arterial vessels such as the mesencephalic and posterior cerebral arteries (e.g. lizards and birds).
Despite these variations, amniotes retain conservation of arterial branching from the PCC/basilar system of vessels, especially the segmental transverse arteries that encircle the hindbrain and give rise to penetrating arteries. 


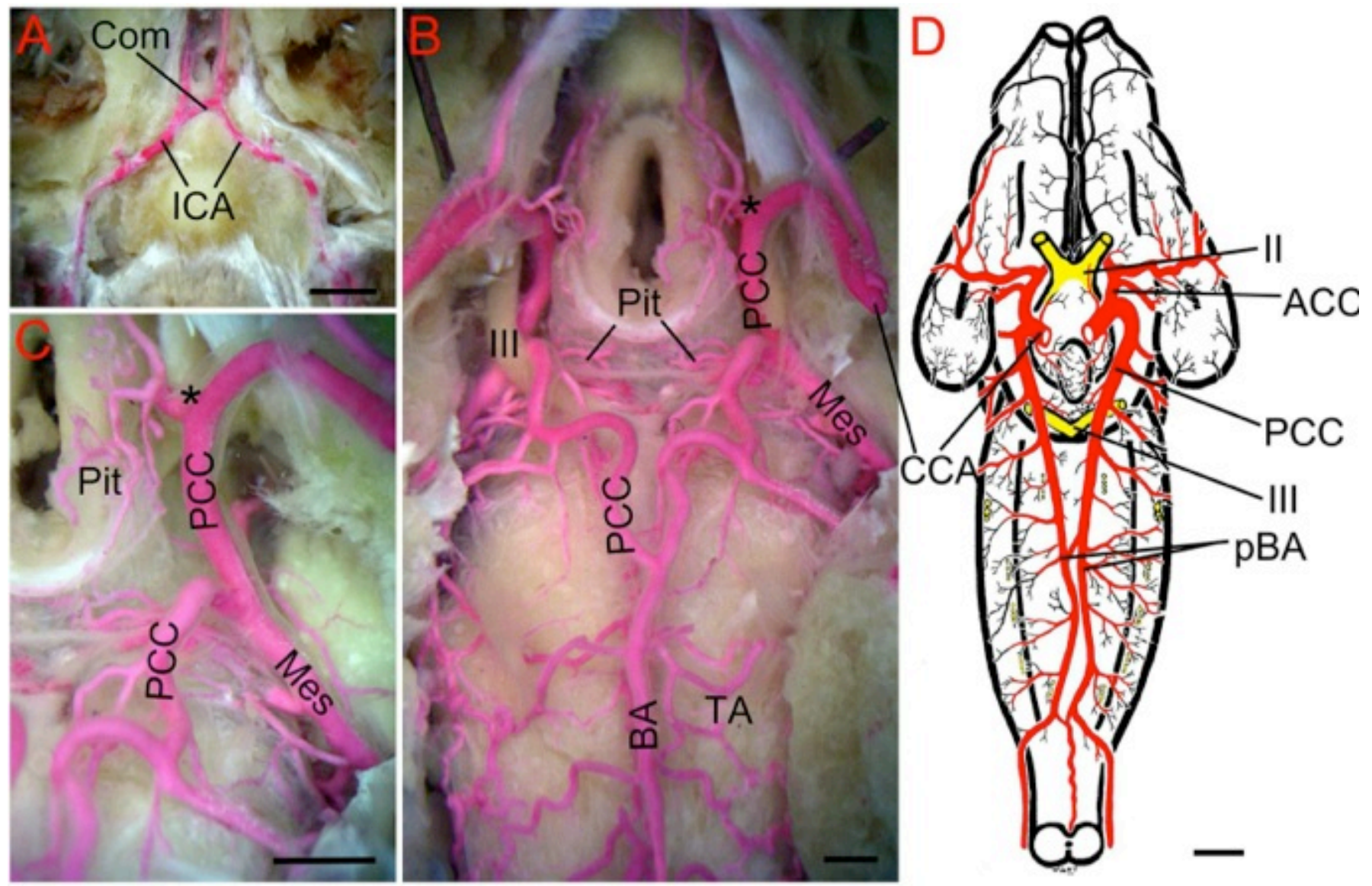

Fig. (10). Turtle. A. Ventral midline view of a red latex-filled Painted Turtle (Chrysemys picta) dissected to show the rostral-directed internal carotid arteries (ICA) forming a short commissural crossbridge (Com) prior to entering the braincase. B. Deeper dissection showing the cerebral carotid arteries (CCA) inside the braincase dividing (marked by black *) into the anterior (ACC; hidden from view) and posterior (PCC) divisions. The PCC give off medially-directed pituitary branches (Pit) and large mesencephalic arteries (Mes) before joining to form a midline unpaired basilar artery (BA). Note the location of this fusion is more caudal than in mammals, but similar to elasmobranchs. The BA gives off a series of rhombencephalic transverse arteries (TA). C. Higher magnification view of the PCC giving off Pit and Mes arteries. D. Brain of Geometric Tortoise (Testudo geometrica) modified after Schepers [78] showing that the PCC continue as paired basilars (pBA) that give off transverse branches. The pBA are connected by small cross-bridges caudal to the trigeminal nerves. II, optic nerve; III, oculomotor nerve. Scale bars are $5 \mathrm{~mm}$ in $\mathrm{A}, 1 \mathrm{~mm}$ in $\mathrm{B}$ and $\mathrm{C}$, and $1 \mathrm{~cm}$ in $\mathrm{D}$.

\section{Chelonia (Turtles)}

The cerebral vessels seen in dissections of injected painted turtles, Chrysemys picta $(\mathrm{N}=2$; Fig. 10), share general features with those of the South African geometric tortoise (Testudo geometrica) described by Schepers [78] and the Galapagos tortoise (Testudo vicina) described by Beddard [79], but differ in morphology of the basilar artery. The paired ICAs in the painted turtle are joined by a crossbridge before entering the skull through carotid canals (Fig. 10A). The CCAs travel adjacent to the hypothalamus and divide into anterior and posterior divisions caudal to the optic chiasm. The PCCs give off pituitary and mesencephalic branches (Fig. 10B, C) before fusing in the midline at the level of the trigeminal nerve roots to form a tortuous unpaired basilar artery (Fig. 10B). While there is sometimes a short cross-bridge just anterior to the oculomotor nerves, the PCCs in T. geometrica (Fig. 10D) and T. vicina [79] run caudally beneath the pons and medulla as separate, paired basilar arteries. In both Chrysemys and Testudo, the basilars give off transverse, circumferential branches to the brainstem and cranial nerve roots. The presence of paired BA in one turtle species and an unpaired basilar in others suggests that developmental studies of different chelonians may provide insights into the primitive patterns of basilar artery organization in amniotes.

\section{Lepidosaurs}

\section{Tuatara}

The tuatara, Sphenodon punctatus, belongs to a relict taxon that is basal to all other living lepidosaurs. The intracranial arteries of the tuatara were described by Dendy [80]. The CCAs enter the cranial cavity on either side of the infundibulum and divide into anterior and posterior branches (Fig. 11A). The ACCs give off vessels that supply the telencephalon and then continue as one of the sources of the "ophthalmic" arteries (Oph). The PCCs give off the mesencephalic arteries (Mes) and then fuse rostral to the trigeminal nerve roots to form an unpaired basilar artery. The BA extends caudally to the roots of the hypoglossal nerve and gives off transverse arteries supplying the hindbrain. Caudal to the hypoglossal roots, the basilar divides into right and left halves before reuniting just behind the roots of the 
$1^{\text {st }}$ spinal nerve. Whether this constitutes a variable basilar fenestration or a proper circulus arteriosus is not known. The presence of an unpaired basilar in the tuatara suggests that paired basilars as seen in Testudo are not primitive for 'reptiles'.

\section{Lizards}

The branching pattern of the cerebral carotid arteries of some lizards is unusual relative to other vertebrates [81]. The internal carotid arteries in lizards enter the intracranial cavity in the typical manner, traveling along the sides of the hypophysis where they divide into anterior and posterior cerebral carotid arteries. However, the location of this division in some lizards (Tupinambis nigropuntatus [79]; Crotaphytus collaris [81]) is much more caudal than in other vertebrates. Likewise unusual is that the mesencephalic arteries in these species branch from the ACCs rather than from the PCCs (Fig. 11B). Some other lizard species (e.g., the skink Eumeces algeriensis [79]) do not have these features but instead branch in the pattern seen in tuatara and most other amniotes. Interestingly, developmental modifications in some lizards are rather abrupt, as the Mes branches in $4.5 \mathrm{~mm}$ embryos of Lacerta muralis come from the PCCs, but arise from the ACCs at $7.8 \mathrm{~mm}$ stages [81]. Burda postulated that the extreme pontine flexure in lizards brings the diencephalon and telencephalon more dorsally than usual, and that the proximal portion of the PCCs is absorbed into the ACCs, moving the final ACCs/PCCs division caudally and yielding an origin of the Mes arteries from the ACCs [81]. Regardless of the position of the $\mathrm{ACCs} / \mathrm{PCCs}$ bifurcation and the origin of the mesencephalic artery, the remainder of the PCCs is similar within lizards, as they extend caudally a short distance and fuse with their opposites to form an unpaired basilar artery. The basilar travels a straight or winding course along the midline on the ventral side of the brain, sends out transverse penetrating branches, connects with the first spinal artery and continues as the ventral spinal artery (Fig. 11B).

\section{Snakes}

The intracranial arteries of a number of snake species have been described by Beddard [79], O'Donoghue [26], Gillilan [82] and others. The general patterns of carotid and basilar branches in snakes are similar to those of lizards (Fig. 11B, C) with the notable difference that one of the common carotid arteries (either left or right) in adult snakes is often reduced or absent $[26,82]$. In the developing grass snake (Tropidonotus natrix) the left and right common carotid arteries arise from the pulmonary aorta and give off the first spinal arteries before dividing into external and internal branches [26]. The blood supply to the brain comes from paired cerebral carotid arteries (CCAs) that arise from the internal carotids, enter the dura near the hypophysis and divide in the pituitary fossa into ACCs, PCCs and Mes, with the latter branch arising variably from either of the former vessels [26]. Variations in the CCA terminus and the Mes origin thus appear to be common in both lizards and snakes. The paired PCCs travel caudally, give off cerebellar

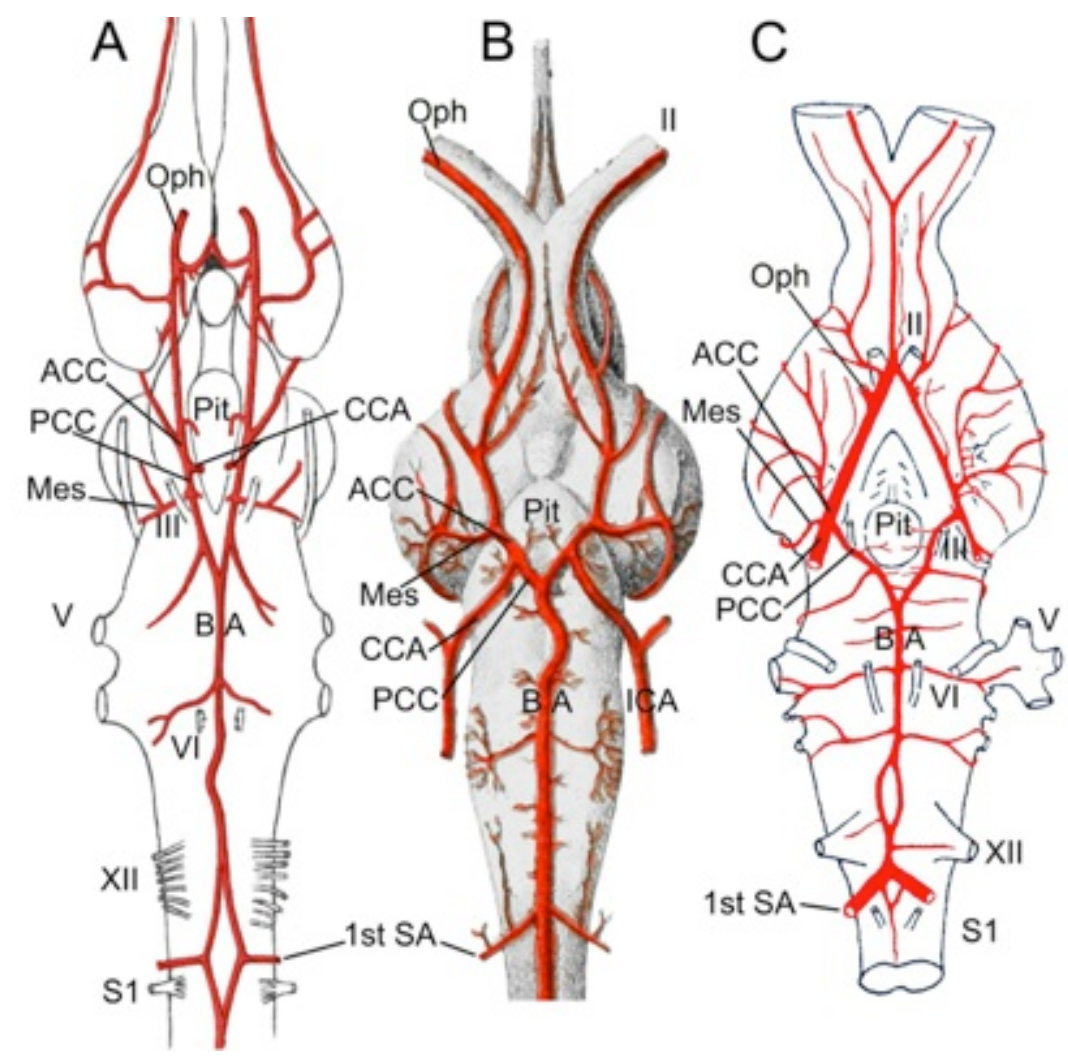

Fig. (11). Lepidosaurs. Ventral views of brain vessels of A) tuatara (Sphenodon punctatus) modified from Dendy [80], B) desert monitor lizard (Varanus griseus) modified from Corti [106] and C) Sonoran lyresnake (Trimorphodon lambda) modified from Gillilan [82] showing variation within lepidosaurs in the branching pattern of CCA into ACC, PCC and Mes divisions. In all three, the PCC fuse to form a midline, unpaired basilar artery (BA) that gives off transverse branches and joins the first spinal artery ( $1^{\text {st }}$ SA) at the spino-medullary junction. Cranial nerve roots are indicated by Roman numerals. Oph, connection of ACC with ophthalmic artery; Pit, pituitary branches. 
branches and unite to form a single midline basilar (BA). The BA gives off transverse branches and joins the first spinal arteries, as in other lepidosaurs. The ACCs travel rostrally along the side of the pituitary, give off ophthalmic and forebrain branches, and unite near the optic chiasm, thus forming a complete arterial ring. The multiple midline anastomoses (ACCs, PCCs, $1^{\text {st }}$ SAs) between the internal carotids of lepidosaurs allow for the degeneration of one common carotid stem in snakes (one among many vascular asymmetries in this group) without disturbing the bilateral symmetry of most carotid-basilar branches [26, 79, 82].

\section{Archosaurs}

Most of the carotid-basilar arterial systems are similar in crocodilians and birds (Fig. 12A-C). However, many avian groups have a unilateral origin of the basilar artery from either the left or right PCCs [83]. Also, the posterior cerebral arteries (PCAs) of birds branch from the ACCs, rather than from the PCCs (Fig. 12C). Crocodilians retain symmetric origins of the PCCs, as well as PCCs origins of the PCAs (Fig. 12A; Caiman latirostris [84], Alligator mississippiensis [85]). The ICAs in crocodilians enter the carotid canal, form a short intercarotid commissure just caudal to the pituitary and then divide into CCAs and ophthalmic branches, with the latter being very large in caimans. The CCAs enter the hypophyseal fossa and divide into paired ACCs and PCCs. The PCCs travel medially and caudally, give off a large caliber PCA and smaller mesencephalic and ventral rostral cerebellar arteries before fusing with their antimeres to form an unpaired midline basilar artery. The basilar gives off segmental circumferential transverse branches before continuing as the ventral spinal artery.

Similar to crocodilians, birds have an intercarotid commissure joining the paired cerebral carotids within the carotid canal (Com, Fig. 12C). The paired CCAs in birds also divide into ACCs and PCCs within the hypophyseal fossa [83]. While a unilateral origin of the PCC/BA is found in most avian groups (Fig. 12C), surprisingly, typical paired basilar stems coming off the PCCs are present in some groups such as galliforms (Fig. 12B), falconiforms and strigiforms [20]. The BA in birds generally gives off transverse vessels to the cerebellum and hindbrain, but not to the mesencephalon, which is supplied by branches from the ACCs, as in some lepidosaurs. Despite these differences in supply to forebrain and midbrain targets, the hindbrain portions of the basilar systems in Archosaurs are similar to those in lepidosaurs and most other amniotes.

\section{Mammals}

The foundation for comparative studies of brain vasculature in mammals was the comprehensive survey published by Hofmann in 1900 [13]. His comparative framework was based on studies of a wide variety of mammals including insectivores, rodents, edentates, carnivores, artiodactyls and perissodactyls [13]. Additional studies on basal mammals [86, 87], primates [15], as well as domesticated and laboratory animals [88-90] have extended knowledge of basic brain vessel anatomy across most of the major branches of the mammalian tree. Ontogenetic studies of brainstem vasculature in mammals are unfortunately very limited, with the classic study of human cerebral arterial development by Padget [14] serving as the basis for interpreting the morphology across the entire class. The elegant SEM study of early cranial arterial development in mice by Hiruma et al. [91] shows that Padget's reconstructions agree in numerous details with other mammals. In particular, the relations between basilar, longitudinal neural and first segmental arteries are very similar in mouse and human. Descriptive studies of brain vessels continue adding to the corpus of mammalian anatomy, often with emphasis on modifications of the carotid and vertebral artery sources [e.g., 92, 93]. These modifications can include reduction or elimination of either the internal carotid or vertebral arteries, along with a variety of anastomotic connections between internal carotid
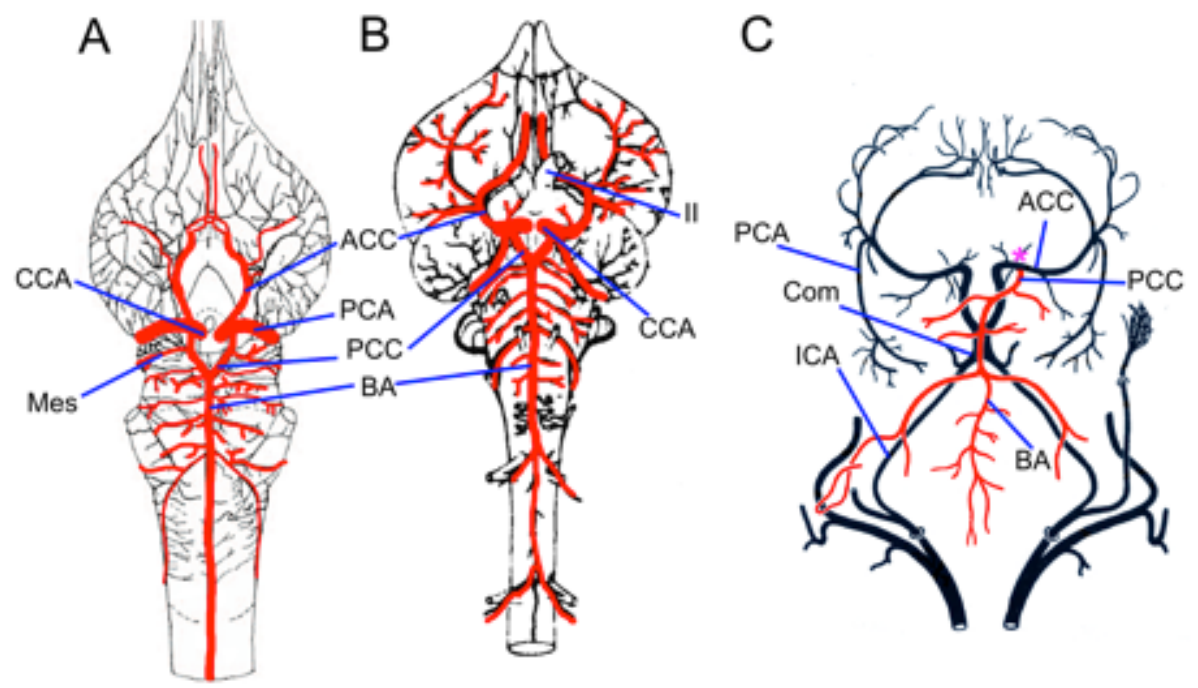

Fig. (12). Archosaurs. Illustrations of a broad-snouted caiman (Caiman latirostris, A) modified from Almeida and Campos [84] and a chicken (Gallus domesticus, B) modified from Hofmann [13] showing the division of the cerebral carotid (CCA) into anterior (ACC) and posterior (PCC) divisions. The PCC fuse to form a midline unpaired basilar artery (BA) that gives off transverse branches. C. Cerebral vessels in a pigeon modified after Baumel [83] showing an unpaired BA arising unilaterally from one PCC, which is typical in most avian groups. The posterior cerebral artery (PCA) arises from the ACC, rather than from the PCC II, optic nerve. 
branches and those of the external carotid, stapedial and other cranial arteries. The microvascular patterns in mammalian brains are also diverse, with marsupials possessing simple arteriovenous loops [94] and other mammals having more or less highly connected anastomotic networks [1]. Despite these differences, the main branches of the posterior cerebral carotid and basilar arteries throughout mammals are nevertheless highly conserved and conform closely to the general pattern for amniotes. The conservative relationship between specific cerebral arterial branches and brain regions in mammals prompted Shellshear [86] to suggest a general principle of neurovascular constancy, where homologous brain nuclei will be supplied by homologous vessels despite large taxonomic differences.

\section{Monotremes (Platypus and Spiny Anteater)}

Shellshear [86] provided a detailed description of the brain vasculature of the spiny anteater, Tachyglossus aculeatus (Fig. 13A). Similar studies of the platypus are not available. The most notable innovation in brain vasculature of the spiny anteater, and presumably of early mammals generally, is the presence of vertebral arteries as additional sources of blood supply for the carotid-basilar system [86]. Essentially all non-mammalian vertebrates receive most of their brainstem blood supply from a rostral to caudal direction through the main CCA/PCC/BA axis [87]. Monotremes are the most basal living group in which blood flows in a caudal to rostral direction through the basilar artery, supplied from the dorsal aorta by way of vertebral arteries. The vertebral arteries join at the level of the uppermost rootlet of CN XII to form an unpaired midline basilar artery. Coming off the vertebral and basilar arteries are circumferential transverse vessels that encircle the pons and medulla. Shellshear [86] described these transverse branches as serially arranged, with the hindbrain thus receiving a segmental arterial supply. The BA in Taychyglossus is similar to that in other mammals as it lies in the groove on the midline of the ventral pons and terminates by dividing into the posterior cerebral arteries (PCA).

\section{Marsupials}

The gross anatomy of the brainstem arteries in the opossum, Didelphis virginiana, is very similar to that described in monotremes [87]. Vertebral arteries join to form an unpaired midline basilar artery, which terminates into posterior cerebral arteries. The vertebral and basilar arteries give off transverse branches that send penetrating arteries into the neuroepithelium. Surprisingly though, the entire brain of the opossum is supplied by penetrating end arteries that terminate as nonanastomotic capillary loops (Fig. 13B) instead of the anastomotic network seen in all other mammals $[1,2,94]$. Each artery enters the pial surface with a corresponding vein (vena comitans) at the same point of entry and they branch together staying paired such that each capillary arteriole and venous venule join to form a slender hairpin loop [94]. This type of brain microvasculature has also been seen in some lungfish, lizards and urodeles [1]. The taxonomic separation between these groups suggests multiple, independent originations of loop vasculature in tetrapods.

\section{Placental Mammals}

Overall, the major arterial pathways supplying the cerebrum and brainstem in placental mammals are highly stereotypical, with most variations involving modifications of the carotid vessels for specialized purposes of thermoregulation and blood flow control [95]. The anatomy of human brain vessels serves as a good general guide for other mammals since the basic pattern of cerebral carotid branches, including connections of the posterior communicating artery with the posterior cerebral (PCA) and basilar arteries, as well as the branches and territories supplied by the basilar and vertebral arteries are relatively unspecialized in humans and are similar in detail to most primates and other generalized placental mammals [13, 15]. In particular, the basic configuration of the Circle of Willis and the positioning of an unpaired basilar artery between the P1 segments of the PCA and the vertebral arteries appear invariant. Likewise, the distribution branches to brainstem regions, possibly including the exact number and locations of segmental pontine branches are also similar between humans and most placental mammals.

The main variations among mammals involve the sources of blood flowing into the carotid-basilar system. Although many vertebrates have anastomotic connections between the basilar artery and branches of the dorsal aorta (e.g., stapedial and hypoglossal arteries), the development of the vertebral artery as a major supply to the basilar is unique to mammals. Since the vascular elements that produce vertebral arteries, namely, intersegmental branches from the dorsal aorta and longitudinal connectors linking them [19], are known to supply the post-cranial axial structures in most vertebrates, the embryonic framework for the emergence of vertebral arteries is nearly ubiquitous. Minor connections linking the longitudinal connector to the basilar artery may also be widespread, not only among amniotes [26, 79] and amphibians [76] but also in teleosts [31] and other fish [22, 75]. It is therefore the magnitude of the caudal connection between the dorsal aorta and basilar artery in mammals, i.e., its functional role, which is unique. Once established, the presence of the vertebral artery as a major brain supply in mammals allows modifications of the carotid supply, including reduction and even elimination of the internal carotid in some groups.

This functional flexibility is further elaborated by supplementing or replacing the carotid supply with retia mirabile stemming from external carotid, stapedial and other arterial pathways [89]. For example, reduction or elimination of the proximal internal carotid is common among carnivores and caviomorph rodents (e.g., capybara and chinchilla). The proximal ICA is reduced in dogs and eliminated in cats, with the maxillary artery supplying the distal ICA in both species through a rete mirabile located in the cavernous sinus $[88$, 89]. While the ICA is similarly absent as an adult structure in chinchillas [96] and capybaras [93], the brain is supplied in these species almost solely by the vertebral arteries. The balance between ICA and vertebral sources can go the other way as well, as shown by the extreme reduction in size of the vertebral arteries in fallow deer [92]. The functional tradeoffs between carotid and vertebral supplies, and the related presence of anastomoses between the ICA and the 

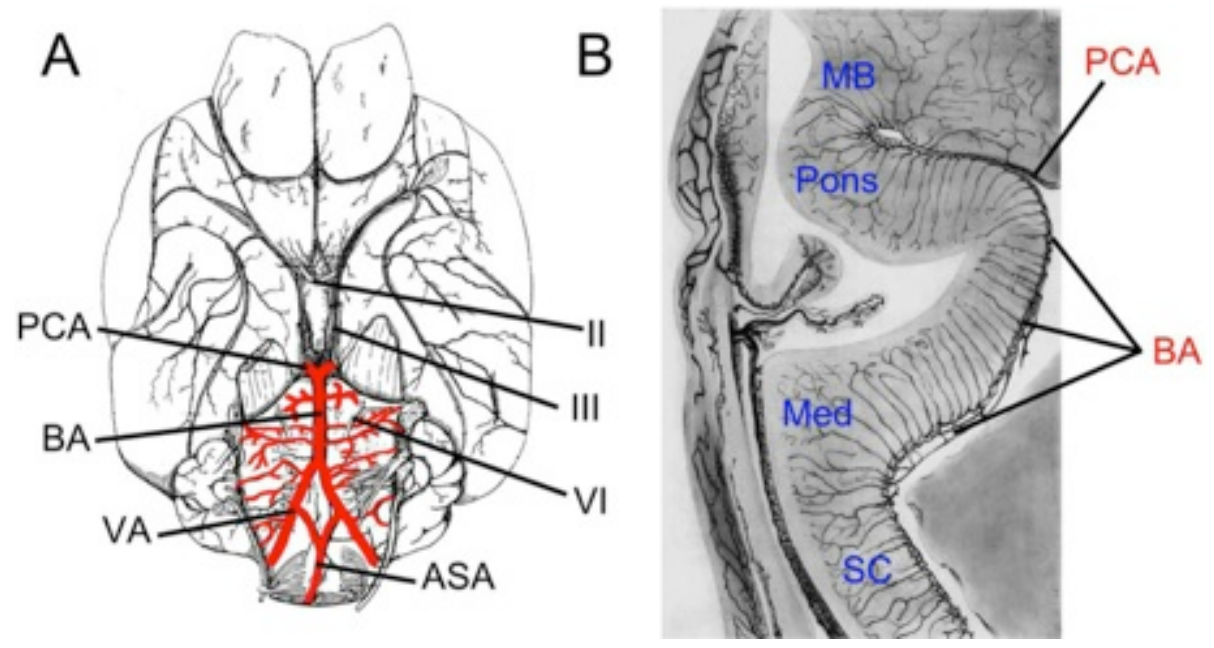

Fig. (13). Monotremes and marsupials: The gross anatomy of cerebral vasculature is similar between the spiny anteater (Tachyglossus aculeatus) and the opossum (Didelphis virginiana). A. Ventral view of a T. aculeatus brain modified after Shellshear [86] showing the caudal to rostral flow of blood as the vertebral arteries (VA) take over as the main hindbrain supply. The VA give off medially-directed commissural branches that form the anterior spinal artery (ASA) and then fuse to form a midline, unpaired basilar artery (BA). The BA gives off numerous transverse branches before terminating as the paired posterior cerebral arteries (PCA) as seen in other mammals. Vertebrobasilar vessels are similar in Didelphis (not shown). B. Sagittal section of an ink-injected opossum brainstem modified after Wislocki [94] showing the BA terminating as the PCA. Note the presence of unique nonanastomotic capillary loops branching from the main cerebral vessels. This differs significantly from the normal capillary network seen in all other mammals.

external carotid or other nearby sources, are evident in the changes that accompany reduction or absence of flow in the human ICAs. Carotid insufficiency can arise by occlusion of patent ICAs or by absence of the ICAs due to agenesis or hypoplasia [97]. Collateral circulation replacing the lost ICAs flow can come by way of the Circle of Willis, either from the vertebral arteries or the contralateral ICAs, if present. Collateral flow can also arise by anastomoses developing with non-ICA vessels such as the maxillary and ascending pharyngeal arteries, or by formation of dense arterial plexi connecting the terminal ICAs to numerous nearby vessels, a condition often called "human carotid rete" $[97,98]$. These tradeoffs are also evident in the development of normal anatomy in humans, where the relative diameters of the P1 segment of the PCA and the junctions of basilar and vertebral arteries vary according to the predominance of flow from either the ICAs or vertebral arteries during fetal development [99].

Given the flexibility in the ultimate source of blood supplying the carotid-basilar system in mammals, the relative constancy of branches distributing to specific regions of the brainstem is all the more notable. This constancy is often masked in older adult animals and humans by the highly tortuous nature of the basilar artery and the asymmetric pattern of transverse basilar branches. Nevertheless, studies of embryonic, fetal and young adult specimens of numerous species show that irregular branching from the basilar and vertebral arteries is not the general case but is likely due to physiological and pathological changes following much more symmetric stages [e.g., 13, 14, 100].

\section{Segmental Basis of Hindbrain Vessels in Mammals}

The transverse vertebro-basilar arterial branches described in young adult rhesus monkey [100] and in developing humans [14] form a ladder-like, quasi-segmental series that, in terms of the relation between transverse vessels and cranial nerve roots, appears to conform closely to the rhombomeric structure of mammalian hindbrains (Fig. 14). For example, the transverse branches from the basilar and vertebral arteries in both an adult rhesus monkey and a Carnegie stage 14 human embryo define a series of segmental divisions starting from the superior cerebellar artery, located at the midbrain-hindbrain junction, and extending back to the middle of the medulla. In both cases, the abducens nerve roots, which are known to develop in rhombomere 5 in mammals [101], are located in the $5^{\text {th }}$ segment of the vascular ladder (Fig. 14A). Likewise, the roots of the trigeminal and facial nerves, known to develop from rhombomeres $2 / 3$ and 4 , respectively, are located laterally in relation to the corresponding divisions defined by the transverse vessels.

In order to further test this correlation, sectioned material from the Carnegie Collection used for the graphic reconstructions in Padget's [14] paper was examined to find stages where both clearly defined rhombomeres (Fig. 14B, C) and the transverse branches of the basilar artery (Fig. 14D) were present. Embryos of Carnegie Stage 14 meet these criteria since the transverse branches begin forming prior to the fusion of the bilateral neural arteries (BNA) to form the midline basilar artery (Fig. 14D), and borders of hindbrain rhombomeres are still evident (Fig. 14B, C). In sagittal sections, rhombomeres 2-6 are visible as broad sulci separated by narrow ridges on the ventricular surface, and as bulges separated by shallow grooves overlying interrhombomeric commissural axon tracts on the pial surface. The transverse hindbrain vessels are visible as small endothelial tubes containing blood cells. Each transverse vessel is located adjacent to the pial surface of the interrhombomeric grooves, and no similar vessels are found on the surface of the rhombomeric bulges (Fig. 14B, C). It therefore appears that transverse hindbrain arteries in 

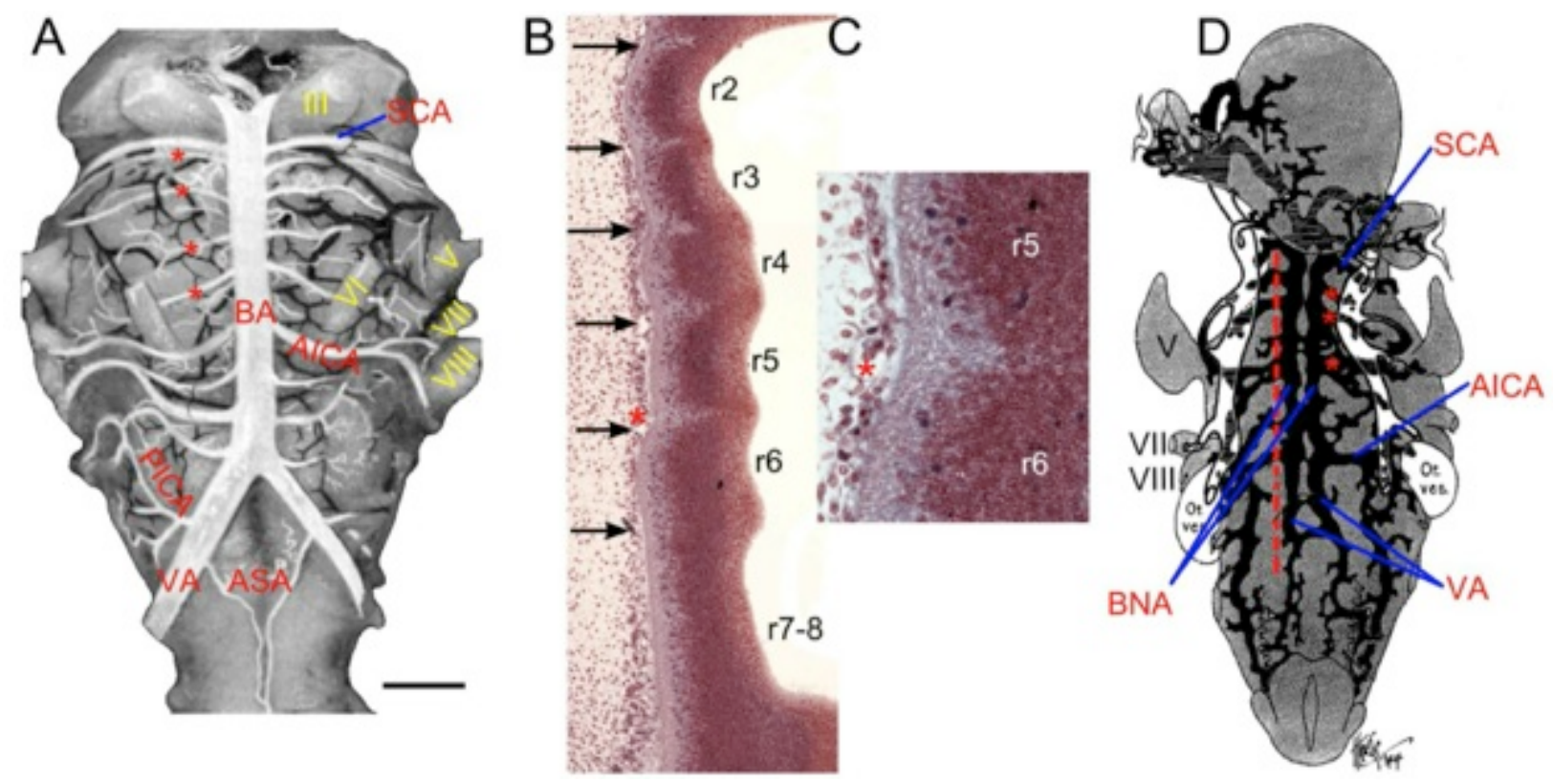

Fig. (14). Rhombomeric transverse branches of basilar. A. The vertebro-basilar arterial pattern of non-human primates is similar to that of humans. This rhesus monkey (Macaca mulatta) hindbrain modified after Gerhard and Olszewski [100] shows a midline unpaired basilar artery (BA) giving off multiple symmetrical transverse branches. The segmental correlation of brain vasculature and neuronal development can be seen as the abducens nerve (VI), located in r5, can also be demonstrated by counting down 4 transverse branches (red asterisks) from the superior cerebellar artery (SCA) to rhombomere 5. B. Sagittal section of Carnegie Stage 14 Human Embryo (\# 9297) showing rhombomere borders and sub-pial vascular elements forming the pontine branches of the basilar artery (arrows). C. Higher magnification of early transverse vessel $\left(^{*}\right)$ lying adjacent to pial surface of r5-6 inter-rhombomeric border. D. Ventral view of graphic reconstruction (modified from Padget [14]) of BA forming by fusion of the primitive bilateral neural arteries (BNA). Transverse arteries (red asterisks) clearly arise prior to this fusion. Red line shows plane of section in B. III, oculomotor nerve; V, trigeminal nerve; VI, abducens nerve; VII, facial nerve; VIII, vestibulocochlear nerve; AICA, anterior inferior cerebellar artery; ASA, anterior spinal artery; PICA, posterior inferior cerebellar artery; VA, vertebral artery.

mammals are truly segmental in origin and form initially along the pial surface of the inter-rhombomeric borders.

\section{DISCUSSION}

\section{General Brainstem Vascular Patterns Across Vertebrates}

Despite evidence that the main inputs to the carotidbasilar system are eminently malleable (e.g., batoids, lepidosaurs, aves, placental mammals), it appears that major distribution branches to the mid- and hindbrain are highly conserved across vertebrates. In short, supply to the brainstem from branches of the PCCs and basilar arteries was seen in all vertebrates examined, regardless of reduction of the internal carotid arteries and emergence of the efferent pseudobranchial arteries, the presence or absence of choroidal and carotid retia, or the dominance of vertebral arteries. Also universal among vertebrates are 1) the ontogenetic origins of the cerebral carotid arteries as rostral extensions of the dorsal aortae, 2) entry of the CCAs into the hypophyseal fossa, 3) bifurcation of the CCAs into cranial and caudal divisions (ACCs and PCCs) just behind the optic nerve, and finally, 4) either fusion of the PCCs to form a single midline basilar, or retention of paired basilar arteries. In the few species whose embryology has been described so far, the single or paired basilar arteries develop from medial migration or sprouting from "primitive lateral vessels" [14, 31]. In most taxa, the developing basilars, whether paired or median, have quasi-segmental transverse branches that extend circumferentially around the brain sending radial penetrating arteries into the neuroepithelium and supplying similar neuronal territories of the brainstem.

The major departures from this general pattern can be readily interpreted as later ontogenetic modifications of the common developmental pattern. These include loss of internal carotids in species such as holocephalans and some mammals as well as the unusual branching sequence of the cerebral carotids in some lizards and snakes. The basic arterial anatomy is retained even in vertebrates whose entire brainstem is supplied by vascular capillary loops, demonstrating at least partial independence between patterning of micro- and macrovasculature. Aside from the almost complete absence of brain vasculature in some salamanders, the most extreme divergence from the common pattern appears to be the restriction of brainstem penetrating vessels to the ventral midline and the consequent almost purely intramural vascular branching seen in teleosts. Some of the variations within vertebrate groups and the unique nature of teleost brain vasculature will be discussed below.

\section{Brainstem Supply from Carotid Arteries and Other Sources}

Although the internal carotid arteries are the primitive and most widespread supply of blood to the brain in vertebrates, cases where internal carotids become reduced or eliminated occur in a number of unrelated taxa. For example, in Chondrichthyes, the efferent pseudobranchial artery can be the dominant (most sharks) or sole (holocephalans; 
anterior carotid) source for brain blood supply, or it can be absent as in Torpedo. In Squalus embryos, the pseudobranchial arteries are significantly larger in diameter than the internal carotids, thus indicating a higher blood flow through these vessels [30, 45]. Nevertheless, despite the variations in the anatomy of pseudobranchial and carotid arteries in cartilaginous, ray-finned and lobe-finned fish, the cerebral carotid and basilar arteries of these species all conform to the general vertebrate pattern. In tetrapods, replacement of parts of the main internal carotid channel is found mainly among amniotes where the vertebral arteries or rete mirabile provide non-carotid sources.

The variability of sources for the basilar system, combined with the relative constancy of its major distribution branches, raises questions about the physiological implications of changing the direction of blood flow in the basilar artery, both on developmental and phylogenetic levels. The fact that cerebral blood flow in different mammals can come exclusively from the internal carotids (fallow deer; [92]) or the vertebrals (capybara; [93]) shows that the basilar artery can adapt to altered directions of flow depending on anatomical conditions. The presence of near right-angled branches off the basilar artery has always been somewhat of a functional enigma, given the smaller branch angles that are found in other parts of vertebrate vasculature. The perpendicular intersections may be what allows blood to be supplied to the brainstem from rostrally (internal carotids) or caudally (vertebrals) without changing the basic branching geometry of the basilar artery. Bidirectional or reversed flow may also occur in species with paired basilars. The differences in branching angles of the side branches of the paired basilars between skates and sturgeons are suggestive of caudal to rostral flow supplied by spinal sources in the former (Figs. 5C, 6A). Few other major arteries with extensive branches are likely to have the ability to maintain normal physiological functions without regard to the direction of blood flow.

\section{Median versus Paired Basilar Arteries}

While overall conservation of hindbrain vascular supply has generally been observed in this study, variations in the main basilar channel are found not only between different major vertebrate clades, but also within them. For example, in Chondrichthyes, sharks possess a midline unpaired basilar artery, while batoids usually have paired basilars (Fig. 5). The presence of single or paired basilar arteries does not alter the primary function of these vessels, which is to supply the rhombencephalon. Also, while some vertebrates have paired basilars that run as separate, parallel vessels (sturgeon), others have paired basilars with multiple crossbridge anastomoses (bowfin, tiger salamander, geometric tortoise). The one significant variation observed across all vertebrates is the occurrence of a fenestrated basilar in species that typically have an unpaired basilar artery (Chondrichthyes, Fig. 5; humans [97, 102]). This widespread shared variation points to a fundamental conserved developmental plan underlying basilar artery morphogenesis, namely, paired basilar precursors showing varying degrees of midline fusion at various hindbrain levels. Embryonic evidence of primordial brainstem vessels across vertebrates shows that having paired hindbrain vessels is a primitive characteristic that can either remain during development or secondarily modify by fusing into one midline vessel [14, 31]. Paired basilars are seen in adult stages of some basal vertebrates such as lampreys as well as in more derived groups such as batoids, paddlefish, sturgeons, and turtles (Fig. 15). They appear as congenital variations in humans [102] and may be one extreme of a spectrum of phenotypes that includes fenestrated basilars and paired basilars joined by multiple cross-bridges.

When plotted on the vertebrate tree, two hypotheses become clear for explaining the presence of paired versus single basilar arteries (Fig. 15). The first hypothesis is that paired basilar arteries are primitive for vertebrates, starting from jawless fishes, and a single, midline basilar appears independently three times: in cartilaginous fishes, teleosts, and tetrapods. Under this hypothesis, batoids, which are cartilaginous fishes, and turtles, which are tetrapods, have reverted to having paired basilar arteries. The second hypothesis is that the midline basilar arises in some common ancestor of extant gnathostomes and is thus a primitive character for cartilaginous, ray-finned and lobed-finned fishes. In this case, most of the paired basilars in gnathostomes represent independent phylogenetic loss or reversal from an ancestral unpaired state. The re-appearance of adult paired basilars could, in some cases, result from truncation of the general ontogenetic pattern at the 'paired basilar stage'. In effect, this would be a 'juvenilization' of the vascular pattern relative to overall sexual maturation [103], with hindbrain vessels forming embryonically the same as in other groups, but failing to undergo the full ancestral ontogenetic trajectory. In this scenario, the presence of other 'juvenile' characters, such as the largely cartilaginous braincase in adult sturgeons, gars and lungfish, would be expected. A second possible explanation for reappearance of adult paired basilars is physiological adaptation: the paired basilars in some taxa might be somehow more optimal than unpaired basilars in supplying the hindbrain. In these cases, other juvenilized characters would not be expected. Batoids fit the profile for this scenario, as they are the only cartilaginous fish with paired basilars and do not possess other obvious truncated developmental characteristics. Since under both hypotheses, the batoid basilar pattern represents either a character reversal or the origin of a new 'type' of paired basilar, this group may be ideal for comparing the relative efficiencies of paired versus median basilar designs. While we favor the first hypothesis, with three independent originations of median basilars and few reversals, the limited evidence so far is not compelling. Testing these hypotheses will require examination of more species, especially in groups like cartilaginous fish and turtles where both basilar configurations are found, and closer evaluation of the anatomical characters to determine how similar and different the median and paired basilars are in different groups.

\section{Dominant Penetrating Arteries}

Unlike most vertebrates where penetrating vessels enter the brainstem from numerous locations along the pial surface, teleosts have a unique system of penetrating arteries that enter only near or at the midline of the mid- and hindbrain in the form of a series of large-caliber segmental stems, arising from the basal communicating, posterior communicating and basilar arteries. The penetrating vessels 


\section{Basilar Artery Dominant Penetrating Arteries

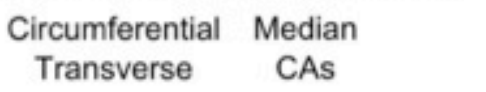

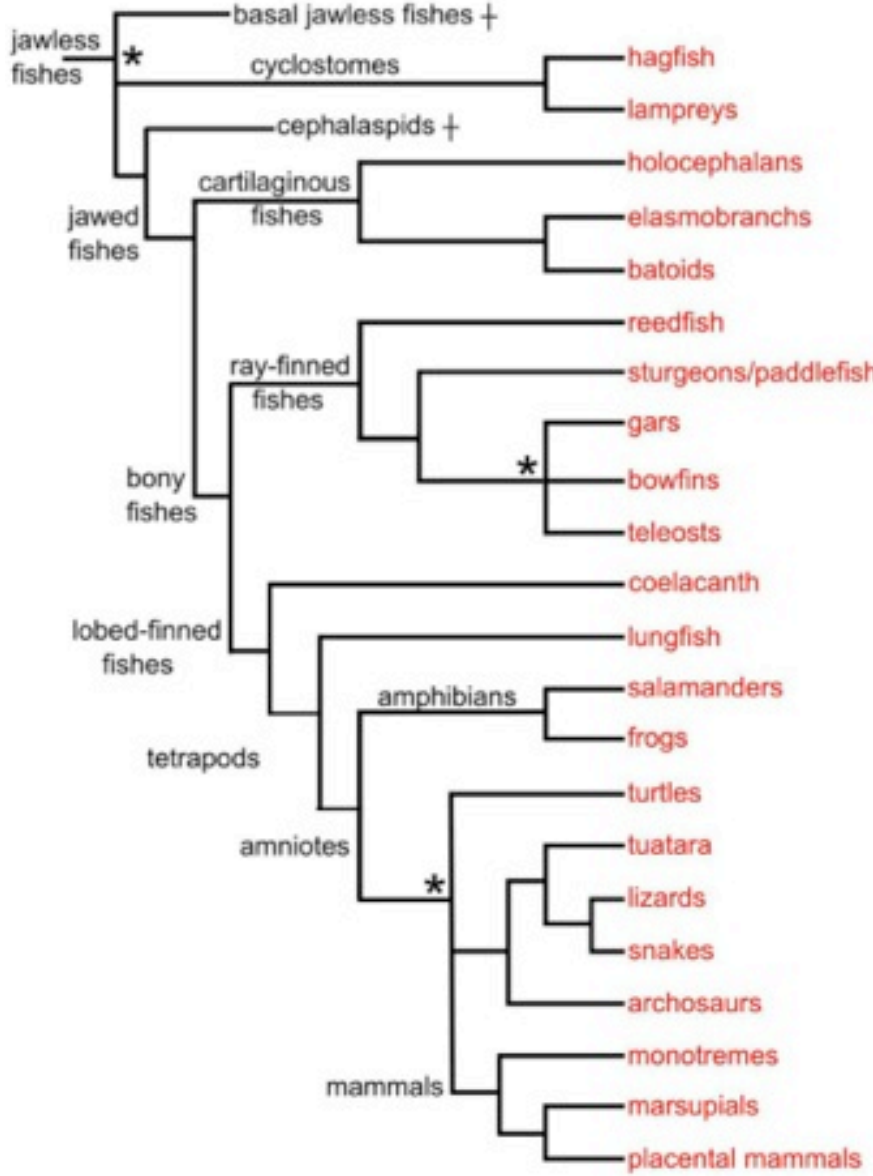
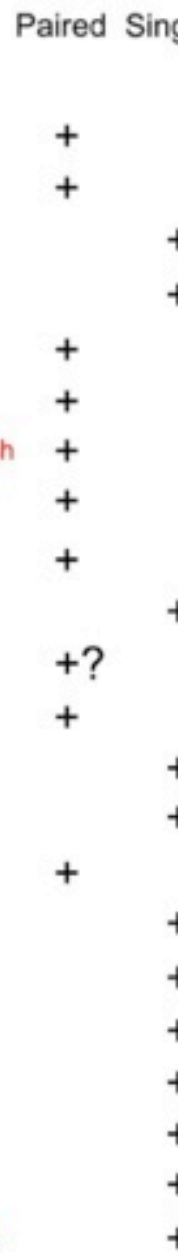

+
+
+
+
+
+
+
+
+
+
+
+
+
+
+
+
+
+
+

Fig. (15). Vertebrate brainstem vascular characters. The presence of paired versus unpaired basilar arteries and the dominance of transverse versus median penetrating arteries are indicated $(+)$ for the different clades examined in this study. Whether the basilar arteries are paired along the entire hindbrain in the coelacanth is uncertain $(+$ ?). Our current hypothesis is that paired basilars are primitive for all vertebrates and that a single basilar arose 3 times independently in cartilaginous fishes, teleosts, and tetrapods. Loss of circumferential transverse arteries and restriction of penetrating branches to midline central arteries is a unique teleost innovation, demonstrating that the neurovascular anatomy of teleosts is clearly derived relative to all other vertebrates.

in teleosts extend towards the subventricular zone and ramify within the neuroepithelium into elaborate intramural vascular trees. This intramural vascular branching and overall ventriculo-pial direction of blood flow contrast greatly with the extramural branching (outside the brain wall) and arterial flow in a pial-ventricular direction with venous drainage in a ventriculo-pial direction seen in other vertebrates. Penetrating branches arising directly from the main basilar channels are present in most or all vertebrates and can be in some cases very numerous and even somewhat segmental in location (e.g., dogfish shark [87]). However, even when highly developed these branches supply only a limited territory near the midline, rather than ramifying bilaterally to supply the entire width of the brainstem as in teleosts. Thus, while the hindbrain central arteries in teleosts play roles similar to the circumferential and penetrating vessels arising from the vertebral and basilar arteries in mammals, their hemodynamic strategy for supplying corresponding neuronal territories is vastly different. Another difference between the unique teleostean hindbrain vascular supply and that of other vertebrates may be the segmental patterning of the main branches of the basilar arteries. While cyprinid fish possess intra-rhombomeric central penetrating arteries that run through the midline of each rhombomere [33], human embryos (Fig. 14), and likely those of most other vertebrates, have transverse arteries that are located at the interrhombomeric borders of each brainstem rhombomere.

\section{Functional and Developmental Mechanisms for Conservation of Carotid-Basilar Anatomy}

The evolutionary persistence of the basic organization of the cerebral carotid and basilar arteries is not due to a lack of developmental or physiological options, as demonstrated by human congenital and pathological cases where flow in major carotid or basilar segments is reduced or eliminated. When the internal carotid arteries become occluded or fail to develop during embryogenesis, the vertebral arteries can increase in size in order to take over as the main blood source to the brain [97]. Variations in the anatomy of other carotid/basilar segments, such as unilateral absence/ degeneration of vertebral, cerebellar or cerebral arteries, along with retention of transitory embryonic vessels such as 
the trigeminal, stapedial and hypoglossal arteries demonstrate the wide range of adaptability of brain vasculature in just one primate species [14, 19, 97, 98]. Similar variations are likely to occur in many species. Along with the dynamic nature of angiogenic and vasculogenic mechanisms known across vertebrates, it seems surprising that a common pattern should exist at all.

With multiple routes for brain blood supply readily accessible in most vertebrate groups, the conservation of basic carotid-basilar anatomy seems likely to result from a combination of functional and developmental constraints. Comparative functional analysis of cerebral blood flow implies being able to measure differences in the efficiency with which alternative vascular designs support neuronal activity. Unfortunately these kinds of studies are almost nonexistent. Ideally, these would require in vivo blood flow measurements and recordings of neuronal activity in intact brains. Short of that, quantitative morphology of the brain vessels along with computer modeling of flow under different anatomical or pressure regimes would be valuable. Some of these types of data are currently used to investigate mechanisms of neurovascular regulation and to develop animal models of stroke [104], but they could also begin to answer whether the conserved carotid-basilar pattern is a more optimal functional solution than the alternatives. The widespread presence of unpaired basilars in major vertebrate crown-groups such as chondrichthyes, teleosts and most major radiations of tetrapods, and the occurrence of paired basilars in more basal lineages, suggest that a single basilar artery may supply blood to the hindbrain in a more efficient manner than paired basilars and might therefore correlate with the generally higher physiological performance of the more derived groups. However, without experimental testing the unpaired basilar as a possible functional innovation for improved neurovascular performance remains purely conjectural.

Wide conservation of adult and embryonic brainstem vessels is also likely to depend on an underlying conserved developmental scaffold. The fundamental segmental patterning of the hindbrain neuroepithelium influences both neuronal, and likely, vascular morphogenesis, thus providing an overall mechanism linking neurovascular structures [33, 101, 105]. The high degree of correspondence between specific neuroepithelial segments and the paths of major carotid-basilar branches suggests that genetic regulatory networks responsible for establishing the brainstem neuronal pattern are either directly or indirectly involved in specifying the matching vascular pattern. Few studies have directly examined genetic pathways that influence hindbrain neurovascular anatomy $[32,33]$, but initial results demonstrate a common segmental basis for integrating neuronal and vascular structures. A conserved toolbox of spatial patterning genes and joint neural-vascular growth regulators should soon begin to reveal a mechanistic basis for Shellshear's hypothesis of neurovascular conservatism [86].

\section{CONCLUSION}

For groups such as lampreys, sturgeons and lungfish, the nascent brainstem vascular framework serves almost directly as a plan for adult brain vasculature, being modified mainly in terms of degree of elaboration of the microvasculature in different species. Thus, hindbrain vascular architecture in some basal vertebrates remains rather rudimentary even when mature, as adults retain paired basilar arteries and a relatively simple vascular network within the brain. Vertebrates higher on the taxonomic tree generally exhibit more elaborate vascular designs, including an unpaired basilar artery and more extensive branching. The high degree of conservation of homologous brain regions, embryonic pharyngeal arch organization and mechanisms of vasculogenesis practically assures that specific brain regions will be supplied by roughly similar arterial pathways across taxa. The conserved vascular design becomes more noteworthy considering the major anatomical, physiological and developmental differences within particular higher taxa, let alone between them. To a first approximation, the proximity of phylogenetic relationships, the disparity in blood sources for the carotid-basilar circulation and the vast divergence of species' lifestyles can largely be ignored when blood flow to specific neuronal systems is being considered, since all taxa examined so far have a brainstem vascular architecture that broadly follows a common plan. The obvious caveat to this is the overall inadequate sample of vertebrate diversity represented by the entire literature on neurovascular anatomy. For the most part, only common and easily accessible, rather than physiologically and ecologically interesting, representatives of major taxa have been examined. Comparisons between direct and indirect developing species, between species with exceptionally large or small body size, and between residents of habitats with high versus low levels of oxygen may well show the limits of the common neurovascular plan and reveal potential mechanisms for circumventing those limits.

\section{CONFLICT OF INTEREST}

The authors confirm that this article content has no conflict of interest.

\section{ACKNOWLEDGEMENT}

Declared none.

\section{REFERENCES}

[1] Craigie EH. Vascular patterns of the developing nervous system. In: Waelsch H, Ed. Biochemistry of the developing nervous system. New York: Academic Press 1955.

[2] Scharrer E. Brain function and the evolution of cerebral vascularization. James Arthur Lectures on the Evolution of the Brain, No. 29. New York: Amer Mus Nat Hist 1960.

[3] De Vriese B. Sur la signification morphologique des artères cérébrales. Arch Biol 1905; 21: 357-457.

[4] Allis E. The pseudobranchial and carotid arteries of the gnathostome fishes. Zool Jahrb Anat Ontog 1908; 27: 103-34.

[5] Allis E. The pseudobranchial and carotid arteries in Esox, Salmo and Gadus, together with a description of the arteries of the adult Amia. Anat Anz 1912; 41: 113-41.

[6] Goodrich ES. Studies on the structure and development of vertebrates. London: Macmillan 1930.

[7] Müller J. Vergleichende Anatomie der Myxinoiden : Dritte Fortsetzung. Über das Gefässsystem. Abhandl Konig Akad Wiss Berlin 1841; pp. 175-303.

[8] Hyrtl J. Das arterielle Gefäßsystem der Rochen. Denkschr Kaiserl Akad Wiss Math-Naturwiss Classe 1858; 15: 1-36.

[9] Allis E. The branchial, pseudobranchial and carotid arteries in Raja radiata. Anat Anz 1912; 41: 579-89.

[10] Grodzinski Z. The main vessels of the brain in rainbow trout. Bull l'Acad Polon Sci Lett Ser B: Sci Nat (II) 1946; Juin 1-19. 
[11] Gaupp E. Anatomie des Frosches. Abt. 2. Lehre vom Nerven-und Gefässsystem. Braunschweig: Friedrich Vieweg und Sohn 1899.

[12] Rathke H. Untersuchungen über die Aortenwurzeln und die von ihnen ausgehenden Arterien der Saurier. Denkschr Kaiserl Akad Wiss Math-Naturwiss Classe 1857; 13: 51-42.

[13] Hofmann M. Zur vergleichenden Anatomie der Gehirn- und Rückenmarksarterien der Vertebraten. Zeitsch Morph Anthro 1900; 2: 247-322.

[14] Padget D. The development of the cranial arteries in the human embryo. Carnegie Inst Wash Publ 575, Contrib Embryol 1948; 32: 205-61.

[15] Duvernoy HM. Human Brainstem Vessels. Berlin: Springer 1978.

[16] De Beer GR. Studies on the vertebrate head. Part I. Fish. Quart J Microsc Sci 1924; 68: 287-341.

[17] Corrington JD. Morphology of the anterior arteries of sharks. Acta Zool 1930; 11: 185-261.

[18] Hochstetter F. Die Entwickelung des Blutgefäßsystems. In: Hertwig O, Ed. Entwickelungslehre der Wirbeltiere. Jena: Gustav Fischer 1906; pp. 21-166

[19] Padget D. Designation of the embryonic intersegmental arteries in reference to the vertebral artery and subclavian stem. Anat Rec 1956; 119: 349-56.

[20] Baumel JJ. Handbook of avian anatomy: nomina anatomica avium. Cambridge, MA: Nuttall Ornithological Club 1993.

[21] Allen W. The blood-vascular system of the Loricati, the mailcheeked fishes. Proc Wash Acad Sci 1905; 7: 27-157.

[22] Grodzinski Z. The blood-vessels in the brain of elasmobranches. Bull l'Acad Polon Sci Lett, Ser B: Sci Nat (II) 1946; 23-85.

[23] Schöbl DJ. Über die Blutgefässe des cerebrospinalen Nervensystems der Urodelen. Arch Mikr Anat 1882; 20: 87-91.

[24] Francis ETB. The anatomy of the salamander. Oxford: Oxford Univ Press 1934.

[25] Moffat DB. The development of the posterior cerebral artery. J Anat 1961; 95: 485-94.

[26] O'Donoghue CH. The circulatory system of the common grass snake (Tropidonotus natrix). Proc Zool Soc Lond 1912; 1912: 61245.

[27] Allis E. The pseudobranchial and carotid arteries in Polyodon spathula. Anat Anz 1911; 39: (257-262; 282-92).

[28] Sterzi G. Die Blutgefasse des Ruckenmarks. Untersuchungen über ihre vergleichende Anatomie und Entwickelungsgeschicte. Anat Hefte 1904; 24: 1-364

[29] Gilland E, Straka H, Baker R and S Zottoli. A hindbrain segmental scaffold specifying neuronal location in the adult goldfish, Carassius auratus. J Comp Neurol 2014; (in press).

[30] Scammon R. Normal plates of the development of Squalus acanthias. Normentafeln Entwickl Wirbel 1911; 12: 1-123.

[31] Isogai S, Weinstein BM. The vascular anatomy of the developing zebrafish: an atlas of embryonic and early larval development. Dev Bio 2001; 230: 278-301.

[32] Fujita M, Cha YR, Pham VN, et al. Assembly and patterning of the vascular network of the vertebrate hindbrain. Development 2011; 138: $1705-15$

[33] Ulrich F, Ma LH, Baker R, Torres-Vasquez J. Neurovascular development in the embryonic zebrafish hindbrain. Dev Biol 2011; 357: 134-51

[34] Janvier P. Early vertebrates. Oxford: Oxford Univ Press 1996.

[35] Delarbre C, Gallut C, Barriel V, Janvier P, Gachelin G. Complete mitochondrial DNA of the hagfish, Eptatretus burgeri: the comparative analysis of mitochondrial DNA sequences strongly supports the cyclostome monophyly. Mol Phylogenet Evol 2002; 22: 184-92.

[36] Jackson CM. An investigation of the vascular system of Bdellostoma dombeyi. J Cinn Soc Nat Hist 1901; 20: 13-48.

[37] Cecon S, Minnich B, Lametschwandtner C. Vascularization of the brains of the atlantic and pacific hagfishes, Myxine glutinosa and Eptatretus stouti: A scanning electron microscope study of vascular corrosion casts. J Morph 2002; 253: 51-63.

[38] Hibbard E. The vascular supply to the central nervous system of the larval lamprey. Am J Anat 1963; 113: 93-9.

[39] Sterzi G. Il sistema nervosa centrale dei vertebrati. Ciclostomi. Padova: Draghi 1907; Vol. 1.

[40] Conel JL. The development of the brain of Bdellostoma stouti. I. External growth changes. J Comp Neurol 1929; 47: 343-403.

[41] Block B, Carey F. Warm brain and eye temperatures in sharks. J Comp Physiol B 1985; 156: 229-36.
[42] Carazzi D. Sul sistema arteriosa di Selache maxima e di altri Squalidi (Acanthias vulgaris, Mustelus vulgaris, Scyllium catalus, S. canicula, Squatina vulgaris). Anat Anz 1905; 26: (63-96: 12434).

[43] Allis E. The pseudobranchial and carotid arteries in Chlamydoselachus anguineus. Anat Anz 1911; 39: 511-9.

[44] Allis E. The branchial, pseudobranchial and carotid arteries in Heptanchus (Notidanus) cinereus. Anat Anz 1912; 41: 478-92.

[45] Sterzi G. Il sistema nervosa centrale dei vertebrati. Pesci. Libro 1: Selaci. Parte 2: Embryologia. Padova: Draghi 1912; Vol. 2.

[46] Muñoz-Chapuli R, Garrido LG. Cephalic blood vessels in elasmobranchs: anatomy and phylogenetic implications. In : Uyeno T, Arai R, Taniuchi T, Matsuura K, Eds. Indo-Pacific fish biology. Tokyo: Ichthy Soc Japan 1986; pp. 164-72.

[47] Norris HW. The plagiostome hypophysis: general morphology and types of structures. Grinnell, Iowa: Science Press Printing Co.; 1941

[48] Daniel JF. The elasmobranch fishes, $2^{\text {nd }}$ ed. Berkeley: Univ California Press 1934

[49] Craigie EH. Notes on the blood vessels of the brain in a Holocephalan, Hydrolagus (Chimaera) colliei. J Comp Neurol 1927; 43: 193-200.

[50] Allis E. The branchial, pseudobranchial and carotid arteries in Chimera (Hydrolagus) colliei. Anat Anz 1912; 42: 10-18.

[51] De Beer GR, Moy-Thomas JA. On the skull of Holocephali. Phil Trans R Soc Lond 1935; 224: 287-312.

[52] Hyrtl J. Kopfarterien der Haifische. Denkschr Kaiserl Akad Wiss Math-Naturwiss Classe 1872; 32: 263-75.

[53] Sterzi G. Il sistema nervosa centrale dei vertebrati. Vol. 2: Pesci. Libro 1: Selaci. Parte 1: Anatomia. Padova: Draghi 1909.

[54] Lauder G, Liem K. The evolution and interrelationships of the actinopterygian fishes. Bull Mus Comp Zool 1983; 150: 95-197.

[55] De Beer GR. The development of the vertebrate skull. Oxford: Oxford Univ Press 1937.

[56] Allis E. The cranial anatomy of Polypterus. J Anat 1922; 56: 189 294.

[57] Gardiner BG. The relationships of the palaeoniscid fishes: a review based on new specimens of Mimia and Moythomasia from the Upper Devonian of Western Australia. Bull Brit Mus Nat Hist 1984; 37: 173-428

[58] Patterson C. The braincase of pholidophorid and leptolepid fishes, with a review of the actinopterygian braincase. Philos Trans R Soc Lond B 1975; 269: 275-579.

[59] Danforth CH. The heart and arteries of Polyodon. J Morph 1912; 23: 409-54.

[60] Demme R. Das Arterielle Gefässsystem von Acipenser ruthenus. Wien: Braumüller 1860

[61] Grodzinski Z. The blood-vessels in the brain of the sturgeon. Bull l'Acad Polon Sci Lett, Ser B: Sci Nat (II) 1948; Septembre, 61-81.

[62] Holmgren N. Studies on the heads of fishes. Part IV. General morphology of the head in fish. Acta Zool 1943; 24: 1-188.

[63] Shearer EM. Studies on the embryology of circulation in fishes. II. Development of the arteries. Am J Anat 1930; 46: 427-59.

[64] Grodzinski Z. Le dévelopement des vaisseaux sanguins dans le cerveau de la truite arc-en-ciel. Bull l'Acad Polon Sci Lett Ser B: Sci Nat (II) 1947; 47-96

[65] von Zwehl V. Uber die Blutgefässversorgung des Gehirns bei eigen Teleostein. Zool Jahrb Abt Anat 1961; 79: 371-438.

[66] Wittenberg JB, Haedrich RL. The choroid rete mirabile of the fish eye. II. Distribution and relation to the pseudobranch and to the swimbladder rete mirabile. Bio Bull 1974; 146: 137-56.

[67] Daeschler EB, Shubin NH, Jenkins FA Jr. A Devonian tetrapodlike fish and the evolution of the tetrapod body plan. Nature 2006 440: 757-63

[68] Ahlberg PE, Johanson Z. Osteolepiformes and the ancestry of tetrapods. Nature 1998; 395: 792-4.

[69] Millot J, Anthony J. Anatomie de Latimeria chalumnae Tome II. Systeme nerveux et organs des sens. Cent Nat Res Sci Paris 1965.

[70] Nieuwenhuys R, Kremers JP, Van Huijzen C. The brain of the crossopterygian fish Latimeria chalumnae: A survey of its gross structure. Anat Embryol 1977; 151:157-69.

[71] Schaeffer B, Gregory JT. Coelacanth fishes from the continental Triassic of the western United States. Amer Mus Novitates No. 2036, 1961; 1-18.

[72] Lagios MD. The pituitary gland of the coelacanth Latimeria chalumnae Smith. Gen Comp Endocrinol 1975; 25: 126-46. 
[73] Spencer WB. Contributions to our knowledge of Ceratodus. Part I. The blood vessels. In: Fletcher JJ, Ed. The Macleay memorial volume. Sydney: Linn Soc NS Wales 1893

[74] Kellicott WE. The development of the vascular and respiratory systems of Ceratodus. Mem NY Acad Sci 1906; 2: 131-249.

[75] Robertson JI. The development of the heart and vascular system of Lepidosiren paradoxa. Q J Microsc Sci 1913; 9: 53-132.

[76] Roofe PG. The endocranial blood vessels of Amblystoma tigrinum. J Comp Neurol 1935; 61: 257-93.

[77] Tsuneki K, Ouji M. Absence of blood vessels of six species of primitive salamanders. Zool Sci 1988; 5: 847-53.

[78] Schepers GW. The blood vascular system of the brain of Testudo geometrica. J Anat 1939; 73: 451-95.

[79] Beddard F. A contribution to the knowledge of the encephalic arterial system in Sauropsida. Proc Zool Soc Lond 1905; 2: 59-70.

[80] Dendy A. The intracranial vascular system of Sphenodon. Philos Trans R Soc Lond B 1909; 200: 403-26.

[81] Burda D. Embryonic modifications of lacertilian intracranial arteries. Am J Anat 1966; 118: 743-54.

[82] Gillilan L. A comparative study of the extrinsic and intrinsic arterial blood supply to brains of submammalian vertebrates. J Comp Neurol 1967; 130: 175-96.

[83] Baumel JJ, Gerchman L. The avian intercarotid anastomosis and its homologue in other vertebrates. Am J Anat 1968; 122: 1-18.

[84] Almeida L, Campos R. A systematic study of the brain base arteries in broad-snouted Caiman (Caiman latirostris). J Morphol Sci 2011; 28: 62-8.

[85] Burda D. Developmental aspect of intracranial arterial supply in the alligator brain. J Comp Neurol 1969; 135: 369-80.

[86] Shellshear JL. A study of the arteries of the brain of the spiny anteater (Echidna aculeata) to illustrate the principles of arterial distribution. Philos Trans Roy Soc Lond B 1930; 218: 1-36.

[87] Gillilan L. Blood supply to primitive mammalian brains. J Comp Neurol 1972; 145: 209-22.

[88] Davis DD, Story HE. The carotid circulation in the domestic cat. Field Mus Nat Hist Zool Series 1943; 28: 5-47.

[89] Gillilan L. Extra- and intra-cranial blood supply to brains of dog and cat. Am J Anat 1976; 146: 237-54.

[90] Scremin OU. Cerebral vascular system. In: Paxinos G, Ed. The rat nervous system, $3^{\text {rd }}$ ed. London: Elsevier 2004; pp. 1165-93.
[91] Hiruma T, Nakajima Y, Nakamura H. Development of pharyngeal arch arteries in early mouse embryo. J Anat 2002; 201: 15-29.

[92] Brudnicki W. Morphometric analysis of the brain base arteries in fallow deer (Dama dama). Veterinarni Med 2011; 56: 462-8.

[93] Steele C, Fioretto E, Sasahara T, Guidi W, de Lima A, Ribeiro A, Loesch A. On the atrophy of the internal carotid artery in capybara. Cell Tissue Res 2006; 326: 737-48.

[94] Wislocki G, Campbell ACP. The unusual manner of vascularization of the brain of the opossum (Didelphys virginiana). Anat Rec 1937; 67: 177-91.

[95] Hébert J, Lust A, Fuller A, Maloney SK, Mitchell D, Mitchell G. Thermoregulation in pronghorn antelope (Antilocapra americana, Ord) in winter. J Exp Biol 2008; 211: 749-56.

[96] Araújo ACP, Campos R. A systematic study of the brain base arteries and their blood supply sources in the chinchilla (Chinchilla lanigera). Braz J Morphol Sci 2005; 22: 221-32.

[97] Grand W, Hopkins L. Vasculature of the brain and cranial base: variations in clinical anatomy. Stuttgart: Thieme 1999.

[98] Yasargil MG. Microneurosurgery. Microsurgical anatomy of the basal cisterns and vessels of the brain. Stuttgart: Georg Thieme Verlag 1984; Vol I.

[99] Van Overbeeke JJ, Hillen B, Tulleken CA. A Comparative study of the circle of Willis in fetal and adult life, The configuration of the posterior bifurcation of the posterior communicating artery. J Anat 1991; 176: 45-54.

[100] Gerhard L, Olszewski J. Medulla oblongata and pons. Primatologia 1969; 2: 3-209.

[101] Gilland E, Baker R. Evolutionary patterns of cranial nerve efferent nuclei in vertebrates. Brain Behav Evol 2005; 66: 234-54.

[102] Goldstein J, Woodcock R, Do H, Phillips C, Dion J. Complete duplication or extreme fenestration of the basilar artery. Am J Neuroradiol 1999; 20: 149-50.

[103] Gould SJ. Ontogeny and phylogeny. Cambridge: Harvard Univ Press 1977.

[104] Bailey E, McCulloch J, Sudlow C, Wardlaw J. Potential animal models of lacunar stroke: a systematic review. Stroke 2009; 40: 451-8.

[105] Ma LH, Gilland E, Bass A, Baker R. Ancestry of motor innervation to pectoral fin and forelimb. Nat Commun 2010; 1: 49.

[106] Corti A. De Systemate Vasorum Psammosauri grisei. Vindobonae: Typis Congregationis Mechistharisticae 1847.

(C) Rahmat and Gilland; Licensee Bentham Open.

This is an open access article licensed under the terms of the Creative Commons Attribution Non-Commercial License (http://creativecommons.org/licenses/by-nc/ 3.0/) which permits unrestricted, non-commercial use, distribution and reproduction in any medium, provided the work is properly cited. 